\title{
The Effect of Online Health Information Seeking on Physician-Patient Relationships: Systematic Review
}

Aijing Luo ${ }^{1,2,3}$, PhD; Lu Qin ${ }^{2,3}$, MS; Yifeng Yuan ${ }^{1,2}$, MS; Zhengzijin Yang ${ }^{2,3}$, MS; Fei Liu ${ }^{2,3}$, MS; Panhao Huang ${ }^{4}$, $\mathrm{PhD}$; Wenzhao Xie ${ }^{2,3}, \mathrm{PhD}$

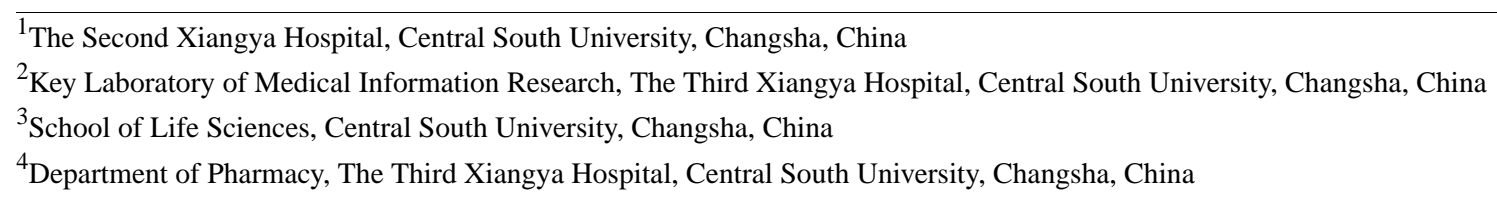

Corresponding Author:

Wenzhao Xie, PhD

Key Laboratory of Medical Information Research

The Third Xiangya Hospital

Central South University

No.138 Tongzipo Road

Changsha, 410013

China

Phone: 86073188618316

Email: xie wenzhao@126.com

\section{Abstract}

Background: The internet has now become part of human life and is constantly changing people's way of life. With the increasing popularity of online health information $(\mathrm{OHI})$, it has been found that $\mathrm{OHI}$ can affect the physician-patient relationship by influencing patient behaviors.

Objective: This study aims to systematically investigate the impact of OHI-seeking behavior on the physician-patient relationship. Methods: Literature retrieval was conducted on 4 databases (Web of Science, PubMed, China National Knowledge Infrastructure, SinoMed), and the time limit for literature publication was before August 1, 2021.

Results: We selected 53 target papers (42 [79\%] English papers and 11 [21\%] Chinese papers) that met the inclusion criteria. Of these, $31(58 \%)$ papers believe that patients' OHI behavior can enable them to participate in their own medical care, improve patient compliance, and improve the physician-patient relationship. In addition, $14(26 \%)$ papers maintain a neutral attitude, some believing that $\mathrm{OHI}$ behavior has no significant effect on doctors and patients and others believing that due to changes in the factors affecting OHI behavior, they will have a negative or a positive impact. Furthermore, 8 (15\%) papers believe that OHI search behavior has a negative impact on doctors and patients, while $6(11 \%)$ papers show that OHI reduces Chinese patients' trust in doctors.

Conclusions: Our main findings showed that (1) OHI-seeking behavior has an impact on patients' psychology, behavior, and evaluation of doctors; (2) whether patients choose to discuss OHI with doctors has different effects on the physician-patient relationship; and (3) the negative impact of OHI on China's internet users is worthy of attention. Due to the low quality of OHI, poor health information literacy, short physician-patient communication time, and various types of negative news, patients' trust in doctors has declined, thus affecting the physician-patient relationship. Improvement of people's health information literacy and the quality of $\mathrm{OHI}$ are important factors that promote the positive impact of $\mathrm{OHI}$ on the physician-patient relationship.

(J Med Internet Res 2022;24(2):e23354) doi: 10.2196/23354

\section{KEYWORDS}

online health information; search behavior; physician-patient relationship; physician-patient consultation. 


\section{Introduction}

About 4.66 billion people worldwide have access to the internet [1]. The internet has gradually become part of human life, constantly changing people's lifestyle. As the availability and immediacy of information services provided by the internet continue to improve, and patients' private information can be concealed to a certain extent, internet health information services have become increasingly attractive [2]. In addition, the lack of medical resources makes people choose to obtain health information online to meet their own health information needs [3]. The online health information (OHI) that patients search for on the internet mainly includes information about diseases, nutrition, treatments, physical and mental health, etc [4,5]. The uneven quality of OHI has a major impact on patients. The credibility of information perceived by patients affects whether the patients use the internet as a frequently used and preferred information source [6]. Physicians are still the most popular source of health information, but the internet has gradually become another important source of health information [7].

OHI seeking can influence physician-patient relationships and patient compliance. Patients who can obtain more health information can better follow the treatment process and enjoy better therapeutic effects [8]. The rapid development of the internet has changed the access of patients to health information and affected the existing physician-patient relationship, which to a large extent determines the medical result that patients receive [9]. As mentioned before, patients choose to obtain health information online to meet their own needs. At the same time, the health information search results provided by the internet show that extreme situations, for example, advice that is contrary to the standard medical opinion or complex data provided by health care professionals, leads to misinterpretation, confusion, and other problems for patients [10]. Of course, the availability of health information on the internet is transforming many patients from passive medical service consumers to those who can participate in the medical process, which brings new challenges for many physicians $[11,12]$. When patients carry a lot of health information in a short consultation, can physicians deal with it as usual?

With the continuous development of the internet, the physician-patient relationship has attracted much attention. In terms of importance, the relationship between patients and physicians is second only to that of family [13]. It is viewed as extremely or very important by $67 \%$, exceeding relationships with spiritual advisors, pharmacists, coworkers, and financial advisors [13]. Due to the patients' lack of understanding of diseases and communication barriers between physicians and patients, some patients cannot understand the results of diagnosis and treatment of the disease and the treatment behavior, which causes a series of problems [14]. Physician-patient communication is a complex clinical behavior whose main goal is to share medical information to improve the education of clinical diagnosis, treatment, and specific diseases [14]. The quality of physician-patient communication affects the physician-patient relationship. In the past, physicians made decisions and patients obeyed them, which constitutes the traditional physician-patient relationship [15]. Patients and medical staff advocate the transition to mutual participation, that is, shared power and responsibility [15]. Previous studies have shown that processing patients' OHI-seeking behavior in daily consultation can improve the quality of medical services [16]. In an ideal physician-patient relationship, patients should be guided instead of looking for $\mathrm{OHI}$ independently [17]. However, at present, patients are mainly looking for OHI by themselves, and they are unable to control the quality of information and other aspects.

In China, the total population is about 1.4 billion [18]. As of June 2021, the number of internet users reached 1.011 billion, and the internet penetration rate reached $71.6 \%$. The "Healthy China" strategy is China's priority development strategy [19]. By implementing internet medicine, the Healthy China strategy promotes the mobility of medical services, enhances the operation efficiency of the overall medical and health system, and optimizes the allocation of medical resources [19]. Due to China's large population and the impact of COVID-19, China's demand for medical resources is growing exponentially. Increasingly more doctors and patients are seeking health information through internet platforms, effectively breaking the time and space restrictions and giving China's unbalanced medical resources a chance to be redistributed [20]. Considering that $\mathrm{OHI}$ may have a positive or a negative impact on the physician-patient relationship, which is important in medical care, this study aims to examine the impact of $\mathrm{OHI}$ on the physician-patient relationship in China.

In recent years, studies on health information seeking have been increasing. It is of great significance to understand the impact of the current health information seeking on the physician-patient relationship. Thus, the purpose of this study is to systematically review the current studies on the impact of $\mathrm{OHI}$ seeking on the physician-patient relationship.

\section{Methods}

\section{Literature Retrieval}

In this study, English references were obtained from the databases Web of Science and PubMed. The PubMed database contains references related to medicine and life sciences. Web of Science includes the most influential core academic journals on natural science, engineering technology, biomedicine, and other research fields. Chinese references were obtained from the databases China National Knowledge Infrastructure (CNKI) and SinoMed. The CNKI is China's largest full-text journal database, while SinoMed focuses on collecting the biomedical literature in China.

After consulting with librarians, the search strategy for this paper consisted of all possible keywords related to 4 topics: (1) online OR internet OR web OR network, (2) wellness information OR health information, (3) search* OR seek* OR inquiry OR query, and (4) physician-patient communication OR doctor-patient communication OR physician-patient relation* OR doctor-patient relation* OR physician-patient interaction OR doctor-patient interaction OR physician-patient trust OR doctor-patient trust. The papers were published before August 31, 2021. Combinations of these keywords were 
searched in the 4 databases to make the literature search as comprehensive as possible. In addition, we searched the PubMed database separately with Medical Subject Headings (MESH). The Mesh terms were "patient-physician relations" and "internet."

This systematic review conforms to the Preferred Reporting Items for Systematic Reviews and Meta-Analyses (PRISMA) statement (Multimedia Appendix 1).

\section{Inclusion and Exclusion Criteria of Publication Standards}

To make the coverage of this study comprehensive enough, the types of papers included were journal papers, conference papers, and academic dissertations. Paper retrieval covered all regions and languages, but only papers with full text in English or Chinese were retained. Papers involving only OHI-seeking studies or only physician-patient relationship studies were excluded. We also excluded all nonempirical research papers, including reviews, research on websites, and research commentaries. Then, we evaluated the quality of the included papers. We used the Critical Appraisal Skills Programme quality assessment tool for qualitative studies, which comprises 10 questions [21] (Multimedia Appendix 2). We also used a quality assessment tool for quantitative studies that comprises 14 questions customized by Tan et al [22] (Multimedia Appendix 3 ). Papers with a quality assessment score lower than 0.7 were excluded.

\section{Paper-Screening Process}

The literature screening in this study was independently carried out by 2 researchers. They screened the titles and abstracts, respectively, and read the full text to extract opinions. We compared the 2 researchers' screening results and the consistency of the extracted views, discussed the discrepancies to ensure the consistency and integrity of results, and used quality assessment tools to assess the quality of the papers. Endnote 20 was used to merge related search results and delete duplicate papers.

\section{Data Extraction and Management}

The research data of papers were independently extracted by 2 researchers according to the predesigned table. It mainly included the following information: country, research design method, sample size, respondents, and conclusion. Where there was ambiguity, the 2 researchers discussed it and reached an agreement.

\section{Results}

\section{Characteristics of the Papers}

In this study, we searched the PubMed and Web of Science databases and retrieved 10,303 and 9345 records, respectively, for a total of 19,648 initially searched records and 15,801 $(80.42 \%)$ exported records that remained after duplication removal using Endnote 20. According to the screening criteria, whether the papers discussed OHI seeking and the physician-patient relationship, 173 (1.09\%) papers were included according to the title and abstract for further screening. Through the application of inclusion and exclusion criteria, of these 173 papers, $72(41.6 \%)$ did not involve the impact of the physician-patient relationship; 7 (4\%) were reviews; for 9 (5.2\%), the original text could not be obtained; 19 (10.9\%) did not have health information seeking as the main research object; $13(7.5 \%)$ had full text in languages other than English; 10 $(5.8 \%)$ were on nonempirical research; and $1(0.6 \%)$ focused on physicians. The screening process is shown in Figure 1.

Finally, we included 42 of 173 (24.2\%) English papers in the study; see Table 1. From the perspective of literature research methods, most of the studies were carried out in the form of questionnaires and interviews, and there were $11(26 \%)$ studies in which research models and hypotheses were first proposed and questionnaires were designed for verification to study the mechanism of OHI seeking affecting the physician-patient relationship from different perspectives [11,23-32]. 
Figure 1. Flowchart of the selection process (English papers). OHI: online health information.

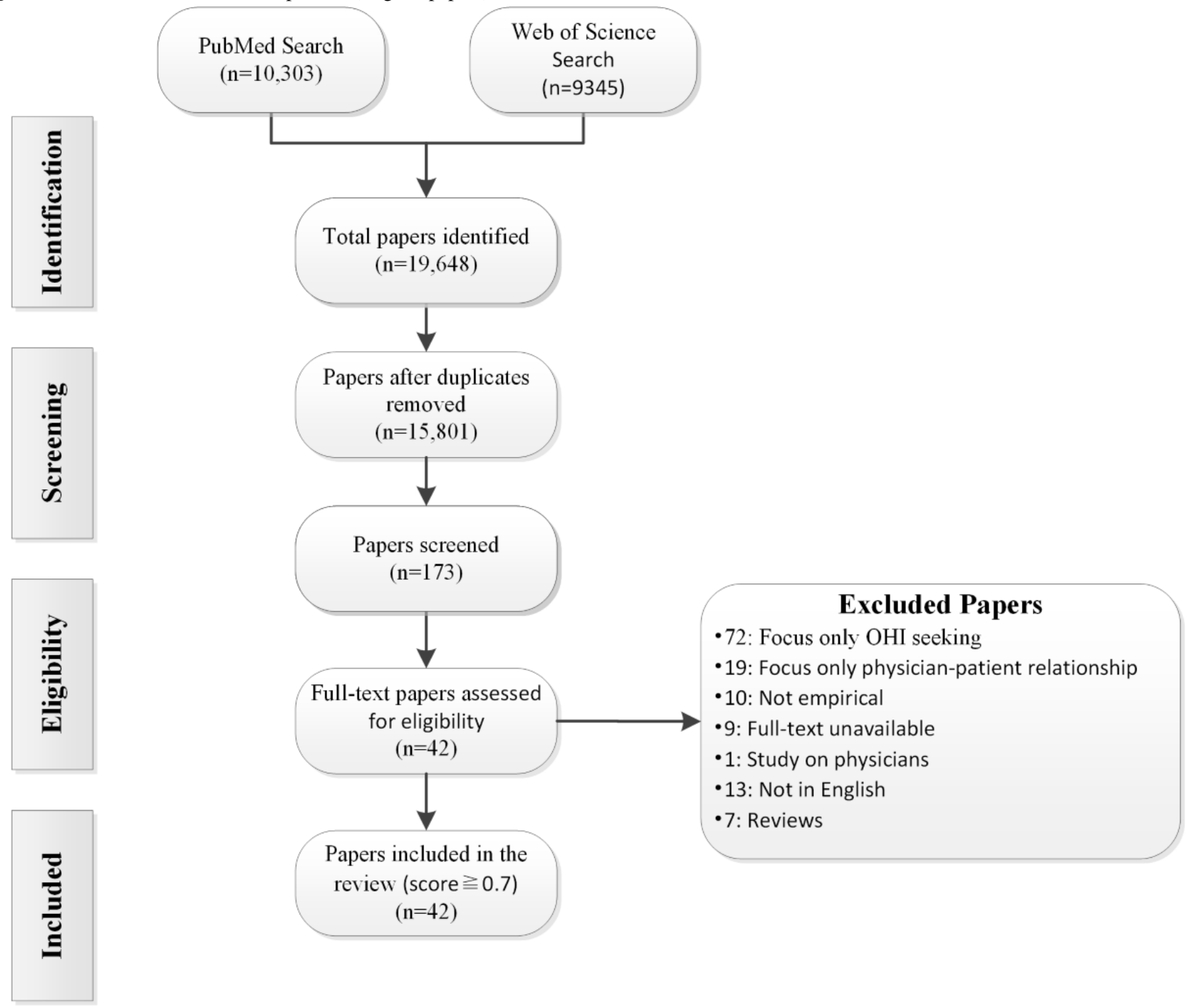


Table 1. Summary of included English papers $(n=42)$.

\begin{tabular}{|c|c|c|c|c|c|c|c|c|c|}
\hline $\begin{array}{l}\text { Num- } \\
\text { ber }\end{array}$ & $\begin{array}{l}\text { Ref- } \\
\text { er- } \\
\text { ence }\end{array}$ & Country & Method & Participants, $\mathrm{n}$ & $\begin{array}{l}\text { Participant charac- } \\
\text { teristics }\end{array}$ & $\begin{array}{l}\text { Factors affect- } \\
\text { ing } \mathrm{OHI}^{\mathrm{a}} \text { use } \\
\text { covered in this } \\
\text { paper } \\
(\mathrm{Y}=\mathrm{yes} / \mathrm{N}=\mathrm{no})\end{array}$ & $\begin{array}{l}\text { Impact of } \\
\text { OHI seeking } \\
\text { on patients } \\
\text { covered in } \\
\text { this paper } \\
\text { (Y=yes/N=no) }\end{array}$ & $\begin{array}{l}\text { OHI seeking af- } \\
\text { fects patients' } \\
\text { evaluation of } \\
\text { physicians cov- } \\
\text { ered in this pa- } \\
\text { per } \\
(\mathrm{Y}=\text { yes/N=no) }\end{array}$ & $\begin{array}{l}\text { Discussion of } \mathrm{OHI} \\
\text { and physician-pa- } \\
\text { tient relationship } \\
\text { covered in this pa- } \\
\text { per ( } \mathrm{Y=yes} / \mathrm{N}=\text { no) }\end{array}$ \\
\hline 1 & [33] & $\begin{array}{l}\text { United } \\
\text { Kingdom }\end{array}$ & $\begin{array}{l}\text { Semistruc- } \\
\text { tured in- } \\
\text { terview }\end{array}$ & $\begin{array}{l}22(12[55 \%] \\
\text { female, } 10 \\
[45 \%] \text { male })\end{array}$ & $\begin{array}{l}\text { Adult patients with } \\
\text { psychosis }\end{array}$ & Y & Y & $\mathrm{N}$ & Y \\
\hline 2 & [7] & Austria & $\begin{array}{l}\text { Email in- } \\
\text { terview }\end{array}$ & $\begin{array}{l}562(332 \\
{[59.1 \%] \text { female, }} \\
230[40.9 \%] \\
\text { male) }\end{array}$ & Internet citizens & $\mathrm{N}$ & $\mathrm{N}$ & $\mathrm{N}$ & Y \\
\hline 3 & [34] & $\begin{array}{l}\text { United } \\
\text { Kingdom }\end{array}$ & Survey & $\begin{array}{l}202(102 \\
{[50.5 \%] \text { female, }} \\
100[49.5 \%] \\
\text { male) }\end{array}$ & $\begin{array}{l}\text { Consecutive adult } \\
\text { hematology clinic } \\
\text { patients }\end{array}$ & Y & Y & Y & $\mathrm{N}$ \\
\hline 4 & [35] & Austria & $\begin{array}{l}\text { Semistruc- } \\
\text { tured in- } \\
\text { terview }\end{array}$ & $\begin{array}{l}26(12[46 \%] \\
\text { female, } 14 \\
[54 \%] \text { male })\end{array}$ & $\begin{array}{l}\text { Patients with } \\
\text { schizophrenia }\end{array}$ & $\mathrm{N}$ & $\mathrm{N}$ & Y & Y \\
\hline 5 & {$[36]$} & $\begin{array}{l}\text { United } \\
\text { States }\end{array}$ & Survey & $\begin{array}{l}154(98 \\
{[63.6 \%] \text { female, }} \\
48[31.2 \%] \\
\text { male, } 8[5.2 \%] \\
\text { missing data) }\end{array}$ & $\begin{array}{l}\text { Patients at } 3 \text { osteo- } \\
\text { pathic primary care } \\
\text { medical clinics }\end{array}$ & $\mathrm{N}$ & Y & $\mathrm{N}$ & Y \\
\hline 6 & [37] & $\begin{array}{l}\text { United } \\
\text { Kingdom }\end{array}$ & $\begin{array}{l}\text { Focus } \\
\text { group in- } \\
\text { terview }\end{array}$ & $\begin{array}{l}34(12[35 \%] \\
\text { female, } 22 \\
[65 \%] \text { male })\end{array}$ & $\begin{array}{l}\text { Adult patients with } \\
\text { diabetes mellitus, } \\
\text { ischemic heart dis- } \\
\text { ease, or hepatitis C }\end{array}$ & $\mathrm{N}$ & $\mathrm{N}$ & $\mathrm{N}$ & Y \\
\hline 7 & {$[38]$} & $\begin{array}{l}\text { United } \\
\text { Kingdom }\end{array}$ & $\begin{array}{l}\text { Email in- } \\
\text { terview }\end{array}$ & $\begin{array}{l}31(28[90 \%] \\
\text { female, } 3[10 \%] \\
\text { male) }\end{array}$ & $\begin{array}{l}\text { Health information } \\
\text { seekers }\end{array}$ & $\mathrm{N}$ & Y & $\mathrm{N}$ & Y \\
\hline 8 & [39] & $\begin{array}{l}\text { Saudi } \\
\text { Arabia }\end{array}$ & Survey & $\begin{array}{l}431(181 \\
{[41.9 \%] \text { female, }} \\
250[58.1 \%] \\
\text { male) }\end{array}$ & $\begin{array}{l}\text { Adult dermatology } \\
\text { outpatients }\end{array}$ & Y & $\mathrm{N}$ & $\mathrm{N}$ & Y \\
\hline 9 & [40] & Canada & $\begin{array}{l}\text { Semistruc- } \\
\text { tured in- } \\
\text { terview }\end{array}$ & $\begin{array}{l}56(30[54 \%] \\
\text { female, } 26 \\
[46 \%] \text { male })\end{array}$ & $\begin{array}{l}\text { Adults aged } \geq 50 \\
\text { years }\end{array}$ & $\mathrm{N}$ & Y & $\mathrm{N}$ & Y \\
\hline 10 & [41] & $\begin{array}{l}\text { United } \\
\text { States }\end{array}$ & $\begin{array}{l}\text { Survey } \\
\text { and } \\
\text { semistruc- } \\
\text { tured in- } \\
\text { terview }\end{array}$ & $\begin{array}{l}120(92 \\
{[76.6 \%] \text { female, }} \\
28[23.3 \%] \\
\text { male) }\end{array}$ & $\begin{array}{l}\text { Patients new to the } \\
\text { rheumatology clin- } \\
\text { ic }\end{array}$ & $\mathrm{N}$ & $\mathrm{N}$ & $\mathrm{N}$ & Y \\
\hline 11 & [42] & $\begin{array}{l}\text { United } \\
\text { States }\end{array}$ & Survey & $\begin{array}{l}70(42[60 \%] \\
\text { recent internet } \\
\text { users [RIUs], } \\
28 \text { [40\%] ever } \\
\text { internet users } \\
\text { [EIUs]) }\end{array}$ & $\begin{array}{l}\text { Breast cancer pa- } \\
\text { tients }\end{array}$ & Y & $\mathrm{N}$ & $\mathrm{N}$ & Y \\
\hline 12 & [43] & $\begin{array}{l}\text { United } \\
\text { States }\end{array}$ & $\begin{array}{l}\text { Survey } \\
\text { and } \\
\text { semistruc- } \\
\text { tured in- } \\
\text { terview }\end{array}$ & $\begin{array}{l}61(49[80 \%] \\
\text { female, } 12 \\
[20 \%] \text { male })\end{array}$ & $\begin{array}{l}\text { New patients with } \\
\text { multiple sclerosis }\end{array}$ & $\mathrm{N}$ & $\mathrm{N}$ & $\mathrm{N}$ & Y \\
\hline 13 & {$[25]$} & $\begin{array}{l}\text { United } \\
\text { States }\end{array}$ & $\begin{array}{l}\text { Individu- } \\
\text { al and fo- } \\
\text { cus group } \\
\text { interview }\end{array}$ & $\begin{array}{l}20(11[55 \%] \\
\text { female, } 9[45 \%] \\
\text { male })\end{array}$ & Older adults & $\mathrm{N}$ & Y & $\mathrm{N}$ & $\mathrm{N}$ \\
\hline
\end{tabular}




\begin{tabular}{|c|c|c|c|c|c|c|c|c|c|}
\hline $\begin{array}{l}\text { Num- } \\
\text { ber }\end{array}$ & $\begin{array}{l}\text { Ref- } \\
\text { er- } \\
\text { ence }\end{array}$ & Country & Method & Participants, $\mathrm{n}$ & $\begin{array}{l}\text { Participant charac- } \\
\text { teristics }\end{array}$ & $\begin{array}{l}\text { Factors affect- } \\
\text { ing } \mathrm{OHI}^{\mathrm{a}} \text { use } \\
\text { covered in this } \\
\text { paper } \\
(\mathrm{Y}=\mathrm{yes} / \mathrm{N}=\mathrm{no})\end{array}$ & $\begin{array}{l}\text { Impact of } \\
\text { OHI seeking } \\
\text { on patients } \\
\text { covered in } \\
\text { this paper } \\
\text { (Y=yes } N=\text { no) }\end{array}$ & $\begin{array}{l}\text { OHI seeking af- } \\
\text { fects patients' } \\
\text { evaluation of } \\
\text { physicians cov- } \\
\text { ered in this pa- } \\
\text { per } \\
(\mathrm{Y}=\text { yes/N=no) }\end{array}$ & $\begin{array}{l}\text { Discussion of OHI } \\
\text { and physician-pa- } \\
\text { tient relationship } \\
\text { covered in this pa- } \\
\text { per }(\mathrm{Y}=\mathrm{yes} / \mathrm{N}=\mathrm{no} \text { ) }\end{array}$ \\
\hline 14 & {$[44]$} & $\begin{array}{l}\text { United } \\
\text { Kingdom }\end{array}$ & $\begin{array}{l}\text { Tele- } \\
\text { phone } \\
\text { survey }\end{array}$ & $\begin{array}{l}3209(1765 \\
{[55 \%] \text { female, }} \\
1444[45 \%] \\
\text { male) }\end{array}$ & $\begin{array}{l}\text { A household proba- } \\
\text { bility sample from } \\
\text { the } 48 \text { contiguous } \\
\text { states }\end{array}$ & $\mathrm{N}$ & $\mathrm{Y}$ & $\mathrm{N}$ & $\mathrm{Y}$ \\
\hline 15 & {$[45]$} & $\begin{array}{l}\text { United } \\
\text { Kingdom }\end{array}$ & $\begin{array}{l}\text { Semistruc- } \\
\text { tured in- } \\
\text { terview }\end{array}$ & $\begin{array}{l}47(32[68 \%] \\
\text { female, } 15 \\
[32 \%] \text { male })\end{array}$ & $\begin{array}{l}\text { Patients with con- } \\
\text { tact with health } \\
\text { services for infor- } \\
\text { mation/treatment } \\
\text { in relation to hor- } \\
\text { mone replacement } \\
\text { therapy } \\
\text { (HRT)/menopause } \\
\text { and Viagra/erectile } \\
\text { dysfunction }\end{array}$ & $\mathrm{N}$ & $\mathrm{N}$ & $\mathrm{N}$ & $\mathrm{Y}$ \\
\hline 16 & [46] & Australia & Survey & $\begin{array}{l}93(44 \text { [47\%] } \\
\text { female, } 49 \\
{[53 \%] \text { male) }}\end{array}$ & Oncology patients & $\mathrm{N}$ & Y & $\mathrm{N}$ & Y \\
\hline 17 & {$[47]$} & $\begin{array}{l}\text { United } \\
\text { Kingdom }\end{array}$ & $\begin{array}{l}\text { Tele- } \\
\text { phone } \\
\text { survey }\end{array}$ & $\begin{array}{l}15 \text { females ( } 8 \\
\text { [53\%] high } \\
\text { school certifi- } \\
\text { cate, } 5[33 \%] \\
\text { bachelor's de- } \\
\text { gree, } 2[14 \%] \\
\text { postgraduate } \\
\text { degree) }\end{array}$ & $\begin{array}{l}\text { Women faced with } \\
\text { decisions concern- } \\
\text { ing menopause and } \\
\text { HRT }\end{array}$ & $\mathrm{N}$ & Y & $\mathrm{N}$ & $\mathrm{N}$ \\
\hline 18 & {$[48]$} & Italy & Survey & $\begin{array}{l}1039(704 \\
{[67.76 \%] \text { fe- }} \\
\text { male, } 335 \\
[32.24 \%] \text { male })\end{array}$ & $\begin{array}{l}\text { Adults aged } \geq 18 \\
\text { years selected from } \\
\text { among parents of } \\
\text { public school stu- } \\
\text { dents }\end{array}$ & $\mathrm{Y}$ & $\mathrm{Y}$ & $\mathrm{N}$ & $\mathrm{N}$ \\
\hline 19 & [49] & $\begin{array}{l}\text { United } \\
\text { States }\end{array}$ & Survey & $\begin{array}{l}5075(3141 \\
{[61.89 \%] \text { fe- }} \\
\text { male, } 1934 \\
[38.11 \%] \text { male })\end{array}$ & $\begin{array}{l}\text { Participants in the } \\
\text { Health Information } \\
\text { National Trends } \\
\text { Survey } 2007\end{array}$ & $\mathrm{Y}$ & $\mathrm{N}$ & $\mathrm{N}$ & $\mathrm{N}$ \\
\hline 20 & {$[50]$} & Australia & Survey & $\begin{array}{l}400(192[48 \%] \\
\text { female, } 208 \\
[52 \%] \text { male })\end{array}$ & $\begin{array}{l}\text { Adult emergency } \\
\text { department pa- } \\
\text { tients }\end{array}$ & $\mathrm{Y}$ & $\mathrm{Y}$ & $\mathrm{N}$ & $\mathrm{N}$ \\
\hline 21 & {$[51]$} & $\begin{array}{l}\text { United } \\
\text { States }\end{array}$ & $\begin{array}{l}\text { Struc- } \\
\text { tured in- } \\
\text { person in- } \\
\text { terview }\end{array}$ & $\begin{array}{l}1142(346 \\
{[30.29 \%] \text { fe- }} \\
\text { male, } 796 \\
[69.71 \%] \text { male })\end{array}$ & $\begin{array}{l}\text { Adults hospitalized } \\
\text { for acute coronary } \\
\text { syndromes }\end{array}$ & $\mathrm{Y}$ & $\mathrm{N}$ & $\mathrm{N}$ & $\mathrm{Y}$ \\
\hline 22 & [9] & Australia & Interview & 33 males & $\begin{array}{l}\text { Patients with } \\
\text { prostate cancer }\end{array}$ & $\mathrm{N}$ & $\mathrm{Y}$ & $\mathrm{N}$ & Y \\
\hline 23 & [10] & $\begin{array}{l}\text { Switzer- } \\
\text { land }\end{array}$ & $\begin{array}{l}\text { Semistruc- } \\
\text { tured in- } \\
\text { terview }\end{array}$ & $\begin{array}{l}32 \text { patients (12 } \\
{[38 \%] \text { female, }} \\
20[62 \%] \text { male) } \\
\text { and } 20 \text { physi- } \\
\text { cians (4 [20\%] } \\
\text { female, } 16 \\
{[80 \%] \text { male) }}\end{array}$ & $\begin{array}{l}\text { Patients and physi- } \\
\text { cians from primary } \\
\text { care and medical } \\
\text { specialist practices }\end{array}$ & $\mathrm{N}$ & $\mathrm{Y}$ & $\mathrm{N}$ & $\mathrm{Y}$ \\
\hline 24 & {$[52]$} & Israel & Survey & $\begin{array}{l}138(83 \\
{[50.7 \%] \text { female, }} \\
54[39.3 \%] \\
\text { male })\end{array}$ & $\begin{array}{l}\text { Patients at } 10 \text { pri- } \\
\text { mary care clinics }\end{array}$ & Y & $\mathrm{Y}$ & $\mathrm{N}$ & $\mathrm{N}$ \\
\hline
\end{tabular}




\begin{tabular}{|c|c|c|c|c|c|c|c|c|c|}
\hline $\begin{array}{l}\text { Num- } \\
\text { ber }\end{array}$ & $\begin{array}{l}\text { Ref- } \\
\text { er- } \\
\text { ence }\end{array}$ & Country & Method & Participants, $\mathrm{n}$ & $\begin{array}{l}\text { Participant charac- } \\
\text { teristics }\end{array}$ & $\begin{array}{l}\text { Factors affect- } \\
\text { ing } \mathrm{OHI}^{\mathrm{a}} \text { use } \\
\text { covered in this } \\
\text { paper } \\
(\mathrm{Y}=\mathrm{yes} / \mathrm{N}=\mathrm{no})\end{array}$ & $\begin{array}{l}\text { Impact of } \\
\text { OHI seeking } \\
\text { on patients } \\
\text { covered in } \\
\text { this paper } \\
\text { (Y=yes } / \mathrm{N}=\text { no) }\end{array}$ & $\begin{array}{l}\text { OHI seeking af- } \\
\text { fects patients' } \\
\text { evaluation of } \\
\text { physicians cov- } \\
\text { ered in this pa- } \\
\text { per } \\
(\mathrm{Y}=\mathrm{yes} / \mathrm{N}=\mathrm{no})\end{array}$ & $\begin{array}{l}\text { Discussion of OHI } \\
\text { and physician-pa- } \\
\text { tient relationship } \\
\text { covered in this pa- } \\
\text { per }(\mathrm{Y}=\mathrm{yes} / \mathrm{N}=\mathrm{no})\end{array}$ \\
\hline 25 & [53] & $\begin{array}{l}\text { United } \\
\text { States }\end{array}$ & $\begin{array}{l}\text { Tele- } \\
\text { phone } \\
\text { survey }\end{array}$ & $\begin{array}{l}2010(1214 \\
{[60.39 \%] \text { fe- }} \\
\text { male, } 796 \\
[39.61 \%] \text { male })\end{array}$ & $\begin{array}{l}\text { Participants in the } \\
\text { Surveying the Dig- } \\
\text { ital Future, Year } 4 \text {, } \\
\text { national survey }\end{array}$ & $\mathrm{N}$ & $\mathrm{N}$ & $\mathrm{Y}$ & $\mathrm{N}$ \\
\hline 26 & {$[54]$} & Canada & Survey & $\begin{array}{l}39(27[70 \%] \\
\text { female, } 11 \\
{[28 \%] \text { male, } 1} \\
[2 \%] \text { unknown })\end{array}$ & $\begin{array}{l}\text { Patients with thy- } \\
\text { roid cancer attend- } \\
\text { ing appointments } \\
\text { with radiation on- } \\
\text { cologists at } 2 \text { ter- } \\
\text { tiary cancer centers }\end{array}$ & $\mathrm{N}$ & $\mathrm{Y}$ & $\mathrm{N}$ & $\mathrm{Y}$ \\
\hline 27 & [12] & $\begin{array}{l}\text { Switzer- } \\
\text { land }\end{array}$ & $\begin{array}{l}\text { Question- } \\
\text { naire sur- } \\
\text { vey }\end{array}$ & $\begin{array}{l}459(207 \\
{[45.1 \%] \text { female, }} \\
252[54.9 \%] \\
\text { male })\end{array}$ & $\begin{array}{l}460 \text { patients aged } \\
\geq 18 \text { years }\end{array}$ & $\mathrm{Y}$ & $\mathrm{N}$ & $\mathrm{N}$ & $\mathrm{Y}$ \\
\hline 28 & {$[26]$} & $\begin{array}{l}\text { Nether- } \\
\text { lands }\end{array}$ & $\begin{array}{l}\text { Question- } \\
\text { naire sur- } \\
\text { vey, in- } \\
\text { terview }\end{array}$ & $\begin{array}{l}90(31[34 \%] \\
\text { female, } 59 \\
[66 \%] \text { male })\end{array}$ & $\begin{array}{l}\text { Patients recently } \\
\text { diagnosed with } \\
\text { colorectal cancer } \\
\text { recruited from } 6 \\
\text { hospitals in the } \\
\text { Netherlands }\end{array}$ & $\mathrm{Y}$ & $\mathrm{Y}$ & $\mathrm{N}$ & $\mathrm{N}$ \\
\hline 29 & {$[55]$} & $\begin{array}{l}\text { United } \\
\text { States }\end{array}$ & $\begin{array}{l}\text { Question- } \\
\text { naire sur- } \\
\text { vey }\end{array}$ & $\begin{array}{l}30(15[50 \%] \\
\text { female, } 15 \\
[50 \%] \text { male })\end{array}$ & $\begin{array}{l}\text { Consecutive pa- } \\
\text { tients presenting } \\
\text { for preoperative } \\
\text { consults for hernia } \\
\text { repair requiring } \\
\text { surgical mesh }\end{array}$ & $\mathrm{Y}$ & $\mathrm{Y}$ & $\mathrm{N}$ & $\mathrm{Y}$ \\
\hline 30 & {$[56]$} & Romania & $\begin{array}{l}\text { Question- } \\
\text { naire sur- } \\
\text { vey }\end{array}$ & $\begin{array}{l}485(242 \\
{[49.9 \%] \text { female, }} \\
\text { mean age } 50.42 \\
\text { years })\end{array}$ & Adult patients & $\mathrm{Y}$ & $\mathrm{Y}$ & $\mathrm{N}$ & $\mathrm{N}$ \\
\hline 31 & [11] & $\begin{array}{l}\text { Singa- } \\
\text { pore }\end{array}$ & $\begin{array}{l}\text { Web- } \\
\text { based } \\
\text { question- } \\
\text { naire sur- } \\
\text { vey }\end{array}$ & $\begin{array}{l}423(209 \\
{[49.4 \%] \text { female, }} \\
214[50.6 \%] \\
\text { male) }\end{array}$ & Internet users & $\mathrm{Y}$ & $\mathrm{N}$ & $\mathrm{N}$ & $\mathrm{Y}$ \\
\hline 32 & [57] & Malaysia & $\begin{array}{l}\text { Question- } \\
\text { naire sur- } \\
\text { vey }\end{array}$ & $\begin{array}{l}381(239 \\
{[62.7 \%] \text { female, }} \\
142[37.3 \%] \\
\text { male) }\end{array}$ & $\begin{array}{l}\text { Patients in a hospi- } \\
\text { tal-based primary } \\
\text { care clinic in the } \\
\text { University of } \\
\text { Malaya Medical } \\
\text { Centre }\end{array}$ & $\mathrm{Y}$ & $\mathrm{N}$ & $\mathrm{N}$ & $\mathrm{Y}$ \\
\hline 33 & {$[58]$} & Belgium & $\begin{array}{l}\text { Qualita- } \\
\text { tive } \\
\text { semistruc- } \\
\text { tured in- } \\
\text { terview }\end{array}$ & $\begin{array}{l}40(22[55 \%] \\
\text { female, } 18 \\
[45 \%] \text { male })\end{array}$ & $\begin{array}{l}\text { Adults between the } \\
\text { ages of } 50 \text { and } 64 \\
\text { years (middle-aged } \\
\text { adults) and } 65 \text { and } \\
80 \text { years (older } \\
\text { adults) }\end{array}$ & $\mathrm{Y}$ & $\mathrm{N}$ & $\mathrm{N}$ & $\mathrm{Y}$ \\
\hline 34 & [59] & China & $\begin{array}{l}\text { Focus } \\
\text { group in- } \\
\text { terview }\end{array}$ & $\begin{array}{l}46(34[74 \%] \\
\text { cancer patients, } \\
12[26 \%] \text { fami- } \\
\text { ly members })\end{array}$ & $\begin{array}{l}\text { Patients with can- } \\
\text { cer or their fami- } \\
\text { lies }\end{array}$ & Y & $\mathrm{Y}$ & $\mathrm{N}$ & $\mathrm{Y}$ \\
\hline
\end{tabular}




\begin{tabular}{|c|c|c|c|c|c|c|c|c|c|}
\hline $\begin{array}{l}\text { Num- } \\
\text { ber }\end{array}$ & $\begin{array}{l}\text { Ref- } \\
\text { er- } \\
\text { ence }\end{array}$ & Country & Method & Participants, $\mathrm{n}$ & $\begin{array}{l}\text { Participant charac- } \\
\text { teristics }\end{array}$ & $\begin{array}{l}\text { Factors affect- } \\
\text { ing } \mathrm{OHI}^{\mathrm{a}} \text { use } \\
\text { covered in this } \\
\text { paper } \\
(\mathrm{Y}=\text { yes } / \mathrm{N}=\text { no) }\end{array}$ & $\begin{array}{l}\text { Impact of } \\
\text { OHI seeking } \\
\text { on patients } \\
\text { covered in } \\
\text { this paper } \\
(\mathrm{Y}=\mathrm{yes} / \mathrm{N}=\mathrm{no})\end{array}$ & $\begin{array}{l}\text { OHI seeking af- } \\
\text { fects patients' } \\
\text { evaluation of } \\
\text { physicians cov- } \\
\text { ered in this pa- } \\
\text { per } \\
(\mathrm{Y}=\text { yes/N=no) }\end{array}$ & $\begin{array}{l}\text { Discussion of } \mathrm{OHI} \\
\text { and physician-pa- } \\
\text { tient relationship } \\
\text { covered in this pa- } \\
\text { per }(\mathrm{Y}=\mathrm{yes} / \mathrm{N}=\mathrm{no})\end{array}$ \\
\hline 35 & [23] & China & Survey & $\begin{array}{l}668(320 \\
{[47.9 \%] \text { preuse }} \\
\text { internet sam- } \\
\text { ples, } 348 \\
{[52.1 \%] \text { not-use }} \\
\text { internet sam- } \\
\text { ples) }\end{array}$ & Internet citizens & $\mathrm{N}$ & Y & $\mathrm{N}$ & $\mathrm{N}$ \\
\hline 36 & [24] & China & Survey & $\begin{array}{l}336(180 \\
{[53.6 \%] \text { female, }} \\
156[46.4 \%] \\
\text { male) }\end{array}$ & $\begin{array}{l}\text { Participants who } \\
\text { underwent treat- } \\
\text { ment with a month }\end{array}$ & $\mathrm{N}$ & $\mathrm{Y}$ & $\mathrm{Y}$ & $\mathrm{N}$ \\
\hline 37 & [27] & China & $\begin{array}{l}\text { Web- } \\
\text { based } \\
\text { question- } \\
\text { naire sur- } \\
\text { vey }\end{array}$ & $\begin{array}{l}336(180 \\
{[53.6 \%] \text { female, }} \\
156[46.4 \%] \\
\text { male) }\end{array}$ & $\begin{array}{l}\text { Chinese individu- } \\
\text { als who received } \\
\text { treatment in the } \\
\text { past month and } \\
\text { searched the inter- } \\
\text { net for health infor- } \\
\text { mation }\end{array}$ & $\mathrm{N}$ & $\mathrm{Y}$ & Y & $\mathrm{N}$ \\
\hline 38 & [28] & China & $\begin{array}{l}\text { Question- } \\
\text { naire sur- } \\
\text { vey }\end{array}$ & $\begin{array}{l}316(194 \\
{[61.4 \%] \text { female, }} \\
122[38.6 \%] \\
\text { male) }\end{array}$ & $\mathrm{OHC}^{\mathrm{b}}$ users & $\mathrm{N}$ & Y & Y & Y \\
\hline 39 & [29] & China & $\begin{array}{l}\text { Web- } \\
\text { based } \\
\text { question- } \\
\text { naire sur- } \\
\text { vey }\end{array}$ & $\begin{array}{l}280(114 \\
{[40.7 \%] \text { female, }} \\
166[59.3 \%] \\
\text { male) }\end{array}$ & $\begin{array}{l}\text { Patients who visit- } \\
\text { ed the hospital } \\
\text { within the past half } \\
\text { year or who are } \\
\text { visiting the doctor } \\
\text { for the first time }\end{array}$ & $\mathrm{N}$ & Y & Y & $\mathrm{N}$ \\
\hline 40 & [60] & $\begin{array}{l}\text { Hong } \\
\text { Kong }\end{array}$ & $\begin{array}{l}\text { Question- } \\
\text { naire sur- } \\
\text { vey }\end{array}$ & $\begin{array}{l}1179(717 \\
{[60.81 \%] \text { fe- }} \\
\text { male, } 462 \\
[39.19 \%] \text { male })\end{array}$ & $\begin{array}{l}\text { Patients attending } \\
\text { the primary care } \\
\text { clinic of a universi- } \\
\text { ty in Hong Kong }\end{array}$ & Y & Y & $\mathrm{N}$ & Y \\
\hline 41 & [31] & China & $\begin{array}{l}\text { Question- } \\
\text { naire sur- } \\
\text { vey }\end{array}$ & $\begin{array}{l}446(224 \\
{[50.2 \%] \text { female, }} \\
222[49.8 \%] \\
\text { male) }\end{array}$ & $\begin{array}{l}\text { Patients in Tongji } \\
\text { Hospital in Wuhan } \\
\text { and the Huazhong } \\
\text { University of Sci- } \\
\text { ence and Technolo- } \\
\text { gy hospital }\end{array}$ & $\mathrm{N}$ & $\mathrm{Y}$ & $\mathrm{Y}$ & $\mathrm{N}$ \\
\hline 42 & [30] & China & $\begin{array}{l}\text { Online } \\
\text { survey }\end{array}$ & $\begin{array}{l}336(180 \\
{[53.6 \%] \text { female, }} \\
156[46.4 \%] \\
\text { male) }\end{array}$ & $\begin{array}{l}\text { Chinese individu- } \\
\text { als who have expe- } \\
\text { rience seeking } \\
\text { health information } \\
\text { and going to hospi- } \\
\text { tals within the pre- } \\
\text { vious month }\end{array}$ & Y & $\mathrm{N}$ & $\mathrm{N}$ & $\mathrm{N}$ \\
\hline
\end{tabular}

${ }^{\mathrm{a} O H I}$ : online health information.

${ }^{\mathrm{b}} \mathrm{OHC}$ : online health community.

With searching in the CNKI and SinoMed, there were 5440 initially searched papers, of which 5219 (95.94\%) exported papers in Chinese remained after duplication removal using Endnote 20. Of these, $88(1.69 \%)$ papers were included according to the title and abstract for further screening. Through the application of inclusion and exclusion criteria, of these 88 papers, $54(61 \%)$ did not involve the impact of the physician-patient relationship; $10(11 \%)$ did not have health information seeking as the main research object; 7 (8\%) were on nonempirical research; for $3(3 \%)$, the original text could not be obtained; and 1 (1\%) focused on physicians. The screening process is shown in Figure 2. All 13 (15\%) papers met the quality rating except for $2(15 \%)$. Finally, 11 of 88 $(13 \%)$ Chinese papers were included in this study; see Table 2. 
The conclusions of these papers were divided into 5 themes: (1) factors that affect patients' use of OHI, (2) the impact of OHI on patients, (3) OHI seeking affecting patients' evaluation of physicians, (4) discussion with physicians about $\mathrm{OHI}$ affecting the physician-patient relationship, and (5) the impact

Figure 2. Flowchart of the selection process (Chinese papers). OHI: online health information.

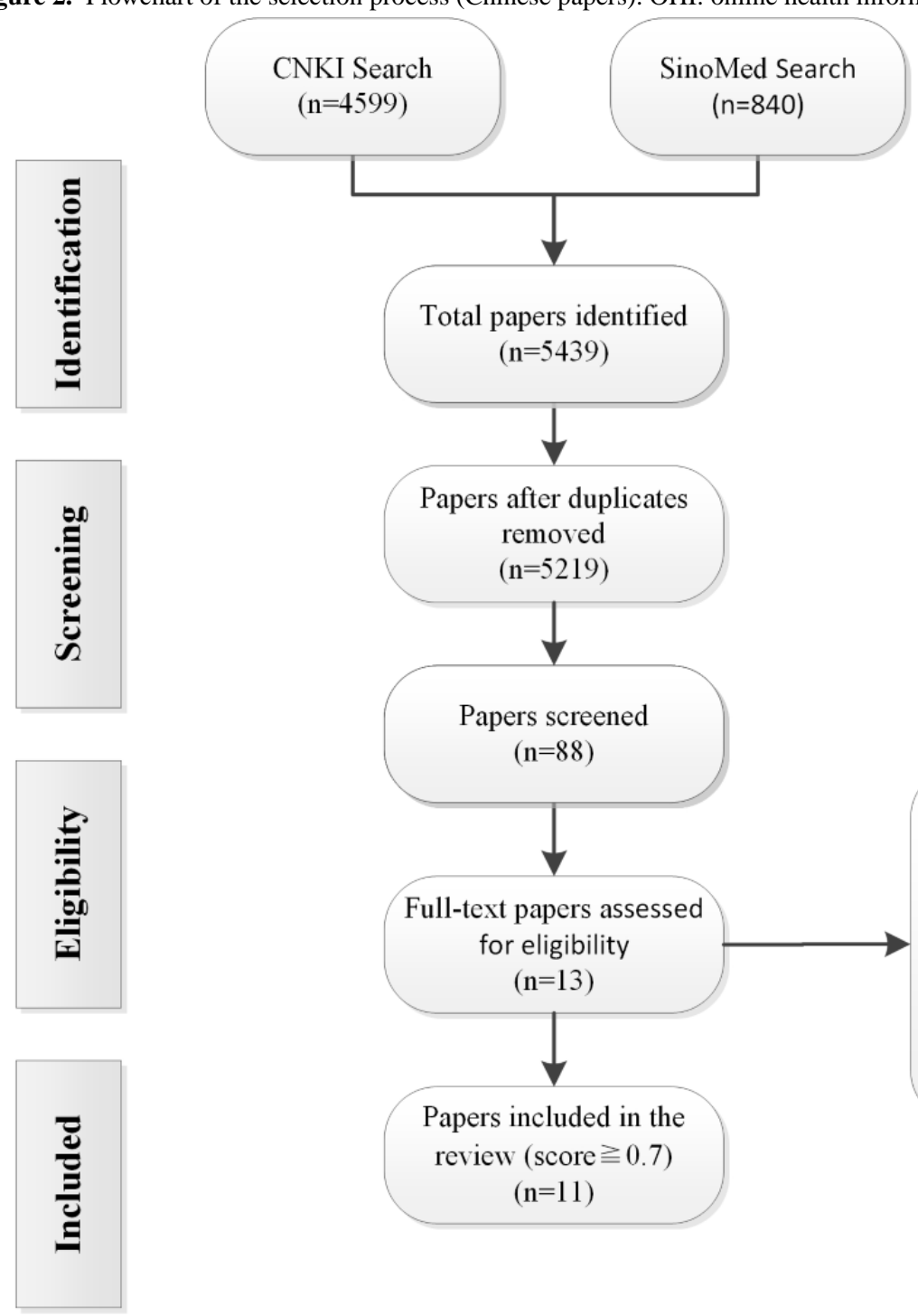

of OHI seeking on the physician-patient relationship, including positive effects, negative effects, and neutral views (Table 3 ). The neutral view refers to no significant effect or both positive and negative effects. 
Table 2. Summary of included Chinese papers $(n=11)$.

\begin{tabular}{|c|c|c|c|c|c|c|c|c|c|}
\hline $\begin{array}{l}\text { Num- } \\
\text { ber }\end{array}$ & $\begin{array}{l}\text { Ref- } \\
\text { er- } \\
\text { ence }\end{array}$ & Country & Method & Participants, $\mathrm{n}$ & $\begin{array}{l}\text { Participant charac- } \\
\text { teristics }\end{array}$ & $\begin{array}{l}\text { Factors affect- } \\
\text { ing } \mathrm{OHI}^{\mathrm{a}} \text { use } \\
\text { covered in this } \\
\text { paper } \\
(\mathrm{Y}=\text { yes/N=no) }\end{array}$ & $\begin{array}{l}\text { Impact of } \\
\text { OHI seeking } \\
\text { on patients } \\
\text { covered in } \\
\text { this paper } \\
(\mathrm{Y}=\text { yes } / \mathrm{N}=\mathrm{no})\end{array}$ & $\begin{array}{l}\text { OHI seeking af- } \\
\text { fects patients' } \\
\text { evaluation of } \\
\text { physicians cov- } \\
\text { ered in this pa- } \\
\text { per } \\
(\mathrm{Y}=\text { yes/N=no) }\end{array}$ & $\begin{array}{l}\text { Discussion of OHI } \\
\text { and physician-pa- } \\
\text { tient relationship } \\
\text { covered in this pa- } \\
\text { per }(\mathrm{Y}=\mathrm{yes} / \mathrm{N}=\mathrm{no})\end{array}$ \\
\hline 1 & {$[61]$} & China & Survey & $\begin{array}{l}179(85 \\
{[47.5 \%] \text { female, }} \\
94[52.5 \%] \\
\text { male) }\end{array}$ & $\begin{array}{l}\text { Outpatients with } \\
\text { chronic diseases }\end{array}$ & $\mathrm{N}$ & $\mathrm{N}$ & $\mathrm{N}$ & $\mathrm{Y}$ \\
\hline 2 & {$[62]$} & China & $\begin{array}{l}\text { Survey, } \\
\text { interview }\end{array}$ & $\begin{array}{l}467(277 \\
{[59.3 \%] \text { female, }} \\
190[40.7 \%] \\
\text { male) }\end{array}$ & Chinese citizens & $\mathrm{Y}$ & $\mathrm{N}$ & $\mathrm{N}$ & $\mathrm{N}$ \\
\hline 3 & {$[63]$} & China & Survey & $\begin{array}{l}446(224 \\
{[50.2 \%] \text { female, }} \\
222[49.8 \%] \\
\text { male })\end{array}$ & $\begin{array}{l}\text { Health information } \\
\text { seekers }\end{array}$ & $\mathrm{N}$ & $\mathrm{Y}$ & $\mathrm{N}$ & $\mathrm{N}$ \\
\hline 4 & [64] & China & Survey & $\begin{array}{l}951 \text { patients } \\
(495[52.1 \%] \\
\text { female, } 456 \\
{[47.9 \%] \text { male) }} \\
\text { and } 888 \text { physi- } \\
\text { cians (348 } \\
{[39.2 \%] \text { female, }} \\
540[60.8 \%] \\
\text { male) }\end{array}$ & $\begin{array}{l}\text { Patients over } 18 \\
\text { and doctors in each } \\
\text { department }\end{array}$ & $\mathrm{Y}$ & $\mathrm{N}$ & $\mathrm{Y}$ & $\mathrm{N}$ \\
\hline 5 & {$[65]$} & China & Survey & $\begin{array}{l}1232(611 \\
\text { [49.59\%] users } \\
\text { of OHI, } 621 \\
\text { [50.41\%] non- } \\
\text { users of OHI) }\end{array}$ & Chinese netizens & $\mathrm{N}$ & $\mathrm{Y}$ & $\mathrm{Y}$ & $\mathrm{Y}$ \\
\hline 6 & {$[66]$} & China & $\begin{array}{l}\text { China } \\
\text { Family } \\
\text { Panel } \\
\text { Studies } \\
\text { (CFPS) } \\
\text { data }\end{array}$ & $\begin{array}{l}29,647(14,815 \\
{[49.97 \%] \text { fe- }} \\
\text { male, } 14,832 \\
[50.03 \%] \text { male })\end{array}$ & Chinese citizens & $\mathrm{Y}$ & $\mathrm{N}$ & $\mathrm{Y}$ & $\mathrm{N}$ \\
\hline 7 & [67] & China & $\begin{array}{l}2013 \text { Chi- } \\
\text { nese So- } \\
\text { cial Sur- } \\
\text { vey data }\end{array}$ & $\begin{array}{l}10,206(2073 \\
{[20.31 \%] \text { neti- }} \\
\text { zens, } 4654 \\
{[45.6 \%] \text { nonneti- }} \\
\text { zens, and } 3479 \\
{[34.09 \%] \text { miss- }} \\
\text { ing data) }\end{array}$ & Chinese citizens & $\mathrm{Y}$ & $\mathrm{N}$ & $\mathrm{Y}$ & $\mathrm{N}$ \\
\hline 8 & [68] & China & Survey & $\begin{array}{l}336(180 \\
{[53.6 \%] \text { female, }} \\
156[46.4 \%] \\
\text { male) }\end{array}$ & $\begin{array}{l}\text { ChunYu Doctors } \\
\text { website users }\end{array}$ & $\mathrm{N}$ & $\mathrm{N}$ & $\mathrm{Y}$ & $\mathrm{N}$ \\
\hline 9 & [69] & China & $\begin{array}{l}2018 \mathrm{CF}- \\
\text { PS adult } \\
\text { question- } \\
\text { naire data }\end{array}$ & $\begin{array}{l}25,015(13,083 \\
{[52.3 \%] \text { female, }} \\
11,932[47.7 \%] \\
\text { male) }\end{array}$ & Chinese citizens & $\mathrm{Y}$ & $\mathrm{N}$ & $\mathrm{N}$ & $\mathrm{N}$ \\
\hline 10 & {$[70]$} & China & $\begin{array}{l}2011 \text { and } \\
2012 \text { Chi- } \\
\text { nese Gen- } \\
\text { eral So- } \\
\text { cial Sur- } \\
\text { vey } \\
\text { (CGSS) }\end{array}$ & $\begin{array}{l}5546 \text { (in 2021) } \\
\text { and } 5797 \text { (in } \\
2012)\end{array}$ & Chinese citizens & $\mathrm{Y}$ & $\mathrm{N}$ & $\mathrm{Y}$ & $\mathrm{N}$ \\
\hline
\end{tabular}




\begin{tabular}{|c|c|c|c|c|c|c|c|c|c|}
\hline $\begin{array}{l}\text { Num- } \\
\text { ber }\end{array}$ & $\begin{array}{l}\text { Ref- } \\
\text { er- } \\
\text { ence }\end{array}$ & Country & Method & Participants, $\mathrm{n}$ & $\begin{array}{l}\text { Participant charac- } \\
\text { teristics }\end{array}$ & $\begin{array}{l}\text { Factors affect- } \\
\text { ing } \mathrm{OHI}^{\mathrm{a}} \text { use } \\
\text { covered in this } \\
\text { paper } \\
(\mathrm{Y}=\mathrm{yes} / \mathrm{N}=\mathrm{no})\end{array}$ & $\begin{array}{l}\text { Impact of } \\
\text { OHI seeking } \\
\text { on patients } \\
\text { covered in } \\
\text { this paper } \\
\text { (Y=yes } / \mathrm{N}=\mathrm{no})\end{array}$ & $\begin{array}{l}\text { OHI seeking af- } \\
\text { fects patients' } \\
\text { evaluation of } \\
\text { physicians cov- } \\
\text { ered in this pa- } \\
\text { per } \\
(\mathrm{Y}=\text { yes/N=no) }\end{array}$ & $\begin{array}{l}\text { Discussion of } \mathrm{OHI} \\
\text { and physician-pa- } \\
\text { tient relationship } \\
\text { covered in this pa- } \\
\text { per }(\mathrm{Y}=\mathrm{ye} / \mathrm{N}=\mathrm{no})\end{array}$ \\
\hline 11 & {$[32]$} & China & $\begin{array}{l}\text { Question- } \\
\text { naire sur- } \\
\text { vey }\end{array}$ & $\begin{array}{l}464(241 \\
{[51.9 \%] \text { female, }} \\
253[48.1 \%] \\
\text { male })\end{array}$ & Chinese citizens & $\mathrm{Y}$ & Y & $\mathrm{Y}$ & $\mathrm{N}$ \\
\hline
\end{tabular}

${ }^{\mathrm{a} O H I}$ : online health information. 
Table 3. $\mathrm{OHI}^{\mathrm{a}}$ seeking affects physician-patient relationships.

\begin{tabular}{|c|c|c|c|c|c|}
\hline \multirow[t]{2}{*}{ Number } & \multirow[t]{2}{*}{ Reference } & \multirow[t]{2}{*}{ Country } & \multicolumn{3}{|c|}{ Impact of OHI seeking on physician-patient relationship } \\
\hline & & & $\begin{array}{l}\text { Positive effects covered in } \\
\text { this paper }(\mathrm{Y}=\mathrm{yes} / \mathrm{N}=\mathrm{no})\end{array}$ & $\begin{array}{l}\text { Neutral views covered in } \\
\text { this paper }(\mathrm{Y}=\mathrm{yes} / \mathrm{N}=\mathrm{no})\end{array}$ & $\begin{array}{l}\text { Negative effects covered in } \\
\text { this paper }(\mathrm{Y}=\mathrm{yes} / \mathrm{N}=\mathrm{no})\end{array}$ \\
\hline 1 & {$[33]$} & United Kingdom & $\mathrm{Y}$ & $\mathrm{N}$ & $\mathrm{N}$ \\
\hline 2 & [7] & Austria & $\mathrm{N}$ & $\mathrm{Y}$ & $\mathrm{N}$ \\
\hline 3 & {$[34]$} & United Kingdom & $\mathrm{Y}$ & $\mathrm{N}$ & $\mathrm{N}$ \\
\hline 4 & {$[35]$} & Austria & Y & $\mathrm{N}$ & $\mathrm{N}$ \\
\hline 5 & {$[36]$} & United States & $\mathrm{Y}$ & $\mathrm{N}$ & $\mathrm{N}$ \\
\hline 6 & {$[37]$} & United Kingdom & Y & $\mathrm{N}$ & $\mathrm{N}$ \\
\hline 7 & {$[38]$} & United Kingdom & $\mathrm{Y}$ & $\mathrm{N}$ & $\mathrm{N}$ \\
\hline 8 & [39] & Saudi Arabia & $\mathrm{Y}$ & $\mathrm{N}$ & $\mathrm{N}$ \\
\hline 9 & [40] & Canada & $\mathrm{N}$ & $\mathrm{Y}$ & $\mathrm{N}$ \\
\hline 10 & {$[41]$} & United States & $\mathrm{N}$ & $\mathrm{Y}$ & $\mathrm{N}$ \\
\hline 11 & [42] & United States & $\mathrm{N}$ & $\mathrm{Y}$ & $\mathrm{N}$ \\
\hline 12 & [43] & United States & $\mathrm{N}$ & $\mathrm{Y}$ & $\mathrm{N}$ \\
\hline 13 & [25] & United States & $\mathrm{Y}$ & $\mathrm{N}$ & $\mathrm{N}$ \\
\hline 14 & {$[44]$} & United Kingdom & $\mathrm{Y}$ & $\mathrm{N}$ & $\mathrm{N}$ \\
\hline 15 & {$[45]$} & United Kingdom & $\mathrm{N}$ & $\mathrm{Y}$ & $\mathrm{N}$ \\
\hline 16 & {$[46]$} & Australia & $\mathrm{Y}$ & $\mathrm{N}$ & $\mathrm{N}$ \\
\hline 17 & [47] & United Kingdom & $\mathrm{Y}$ & $\mathrm{N}$ & $\mathrm{N}$ \\
\hline 18 & [48] & Italy & $\mathrm{Y}$ & $\mathrm{N}$ & $\mathrm{N}$ \\
\hline 19 & [49] & United States & $\mathrm{N}$ & $\mathrm{Y}$ & $\mathrm{N}$ \\
\hline 20 & {$[50]$} & Australia & $\mathrm{Y}$ & $\mathrm{N}$ & $\mathrm{N}$ \\
\hline 21 & {$[51]$} & United States & $\mathrm{Y}$ & $\mathrm{N}$ & $\mathrm{N}$ \\
\hline 22 & [9] & Australia & $\mathrm{N}$ & $\mathrm{Y}$ & $\mathrm{N}$ \\
\hline 23 & {$[10]$} & Switzerland & $\mathrm{N}$ & $\mathrm{Y}$ & $\mathrm{N}$ \\
\hline 24 & {$[52]$} & Israel & $\mathrm{Y}$ & $\mathrm{N}$ & $\mathrm{N}$ \\
\hline 25 & {$[53]$} & United States & $\mathrm{Y}$ & $\mathrm{N}$ & $\mathrm{N}$ \\
\hline 26 & {$[54]$} & Canada & $\mathrm{N}$ & $\mathrm{Y}$ & $\mathrm{N}$ \\
\hline 27 & [12] & Switzerland & $\mathrm{N}$ & $\mathrm{Y}$ & $\mathrm{N}$ \\
\hline 28 & [26] & Netherlands & $\mathrm{N}$ & $\mathrm{Y}$ & $\mathrm{N}$ \\
\hline 29 & {$[55]$} & United States & $\mathrm{N}$ & $\mathrm{N}$ & $\mathrm{Y}$ \\
\hline 30 & {$[56]$} & Romania & $\mathrm{Y}$ & $\mathrm{N}$ & $\mathrm{N}$ \\
\hline 31 & {$[11]$} & Singapore & $\mathrm{Y}$ & $\mathrm{N}$ & $\mathrm{N}$ \\
\hline 32 & {$[57]$} & Malaysia & $\mathrm{N}$ & $\mathrm{N}$ & $\mathrm{Y}$ \\
\hline 33 & [58] & Belgium & $\mathrm{Y}$ & $\mathrm{N}$ & $\mathrm{N}$ \\
\hline 34 & [59] & China & $\mathrm{Y}$ & $\mathrm{N}$ & $\mathrm{N}$ \\
\hline 35 & {$[23]$} & China & $\mathrm{N}$ & $\mathrm{Y}$ & $\mathrm{N}$ \\
\hline 36 & {$[24]$} & China & $\mathrm{Y}$ & $\mathrm{N}$ & $\mathrm{N}$ \\
\hline 37 & [27] & China & $\mathrm{Y}$ & $\mathrm{N}$ & $\mathrm{N}$ \\
\hline 38 & {$[28]$} & China & $\mathrm{Y}$ & $\mathrm{N}$ & $\mathrm{N}$ \\
\hline 39 & [29] & China & $\mathrm{Y}$ & $\mathrm{N}$ & $\mathrm{N}$ \\
\hline 40 & {$[60]$} & China & $\mathrm{N}$ & $\mathrm{N}$ & $\mathrm{Y}$ \\
\hline
\end{tabular}




\begin{tabular}{|c|c|c|c|c|c|}
\hline \multirow[t]{2}{*}{ Number } & \multirow[t]{2}{*}{ Reference } & \multirow[t]{2}{*}{ Country } & \multicolumn{3}{|c|}{ Impact of OHI seeking on physician-patient relationship } \\
\hline & & & $\begin{array}{l}\text { Positive effects covered in } \\
\text { this paper }(\mathrm{Y}=\mathrm{yes} / \mathrm{N}=\mathrm{no})\end{array}$ & $\begin{array}{l}\text { Neutral views covered in } \\
\text { this paper }(\mathrm{Y}=\mathrm{yes} / \mathrm{N}=\mathrm{no})\end{array}$ & $\begin{array}{l}\text { Negative effects covered in } \\
\text { this paper }(\mathrm{Y}=\mathrm{yes} / \mathrm{N}=\mathrm{no})\end{array}$ \\
\hline 41 & {$[31]$} & China & $\mathrm{Y}$ & $\mathrm{N}$ & $\mathrm{N}$ \\
\hline 42 & {$[30]$} & China & $\mathrm{Y}$ & $\mathrm{N}$ & $\mathrm{N}$ \\
\hline 43 & {$[61]$} & China & $\mathrm{Y}$ & $\mathrm{N}$ & $\mathrm{N}$ \\
\hline 44 & {$[62]$} & China & $\mathrm{N}$ & $\mathrm{N}$ & $\mathrm{Y}$ \\
\hline 45 & {$[63]$} & China & $\mathrm{N}$ & $\mathrm{Y}$ & $\mathrm{N}$ \\
\hline 46 & {$[64]$} & China & $\mathrm{Y}$ & $\mathrm{N}$ & $\mathrm{N}$ \\
\hline 47 & [65] & China & $\mathrm{Y}$ & $\mathrm{N}$ & $\mathrm{N}$ \\
\hline 48 & [66] & China & $\mathrm{N}$ & $\mathrm{N}$ & $\mathrm{Y}$ \\
\hline 49 & [67] & China & $\mathrm{N}$ & $\mathrm{N}$ & $\mathrm{Y}$ \\
\hline 50 & [68] & China & $\mathrm{Y}$ & $\mathrm{N}$ & $\mathrm{N}$ \\
\hline 51 & [69] & China & $\mathrm{N}$ & $\mathrm{N}$ & $\mathrm{Y}$ \\
\hline 52 & [70] & China & $\mathrm{N}$ & $\mathrm{N}$ & $\mathrm{Y}$ \\
\hline 53 & [32] & China & $\mathrm{Y}$ & $\mathrm{N}$ & $\mathrm{N}$ \\
\hline
\end{tabular}

${ }^{\mathrm{a} O H I}$ : online health information.

\section{Factors Affecting the Use of $\mathrm{OHI}$}

Studies show that education level, income, gender, age, health literacy, culture, and other factors can affect people's use of OHI $[12,26,33,34,39,42,49,51,56]$. Five papers showed that users with high education level and high income are more willing to use OHI $[12,33,34,39,42,62]$. This population has relatively high health literacy and can better deal with OHI. Patients with difficulties in understanding health information are less likely to ask questions or seek guidance during consultation. Gantenbein et al [12] found that women are more willing to conduct OHI searches, while Aref-Adib et al [33] found that young male psychiatric patients are more likely to discuss health information with their physicians. De Looper et al [26] and Drug et al [56] found that younger patients engage more in OHIS, but Waring et al [51] did not observe the age difference possibly because the large age-grouping scope could not reflect the difference between the elderly and the young. In addition to personal factors, Chiu et al [59] found that the cultural environment of patients may also affect the communication on health factors. In a hierarchical culture of patients and physicians, patients are unwilling to ask questions for fear that the physicians would be unhappy. Instead, they choose to listen to the advice of physicians [59]. In this paper, we only included papers studying the relationship between physicians and patients; however, maybe many other factors also affect the use of $\mathrm{OHI}$ by patients.

\section{Impact of OHI Seeking on Patients}

Several studies have shown that OHI can enhance the communication ability and decision-making ability of patients. The study conducted by Iverson et al [36] showed that $46 \%$ of patients said they would change their health-related behaviors after searching for health information online. After searching the health information online, patients have a certain understanding of their own health status and disease treatment and can better understand the medical terms used by physicians when talking with them [25,38,59]. Murray et al [44] showed that people who discuss health information with physicians often have higher self-assessment ability to assess their own health. Liang et al [61] showed that patients who think that OHI is important and helpful to health decision making are more inclined to think that it will be beneficial to the physician-patient relationship.

However, some patients may show some negative effects after $\mathrm{OHI}$ query. Aref-Adib et al [33] found that some patients may have concerns over what they read and that they change medication adherence and behavior without communication with the physicians. Another concern raised by $\mathrm{OHI}$ seeking is related to the quality of $\mathrm{OHI}$, such as the credibility and limitations of information [40]. OHI will affect patients' decision making, but patients still regard physicians as the main source of health information [47]. Due to the uneven quality of $\mathrm{OHI}$ and the lack of quality control, in addition to patients lacking medical information literacy, the judgments made by patients based on $\mathrm{OHI}$ are generally unscientific and difficult to be recognized by doctors, which may have a negative impact on the physician-patient relationship [62].

\section{OHI Seeking Affects Patients' Evaluation of Physicians}

The impact of $\mathrm{OHI}$ is mainly reflected in patients' trust in and satisfaction with physicians [42]. Patients' satisfaction with physicians is composed of many factors, among which the the main influencing factors are related to the actual communication between patients and physicians [23]. However, most patients are afraid to challenge their doctors, so they are reluctant to discuss their OHI [41]. Patients' satisfaction with OHI has a direct and positive impact on psychological safety [34], while psychological safety might have a direct and positive impact on patients' trust in physicians [24]. When patients use the network health community, the trust relationship among 
community members also affects patients' trust in and satisfaction with physicians [71]. Liu [68] showed that continuous use of online health communities (OHCs) increases users' satisfaction with medical services.

\section{Discussion With Physicians About Health Information Affects Physician-Patient Relationships}

After OHI seeking, some patients choose to share health information with their physicians. Part of the motivation for discussing health information with physicians is that patients want to meet their psychological and emotional needs [42]. Iverson et al [36] showed that $73 \%$ of patients like to discuss $\mathrm{OHI}$ with physicians mainly because they think physicians are willing to discuss OHI. The willingness of physicians to discuss health information with patients is crucial. After discussing their concerns about OHI with physicians, the patients' medication adherence and behavior remain unchanged and the anxiety caused by OHI reduces [33]. In addition, after discussing OHI with physicians, patients' satisfaction with physicians significantly improves [39]. People who discuss OHI with their physicians think it has a positive impact on the disease and their relationship with their physicians [7].

However, some studies have also proved that the main reason patients do not actively discuss health information with physicians is the fear of challenging physicians' authority $[33,35,38,40,42,43,59]$. Patients worry that when they talk about $\mathrm{OHI}$, or express some opinions that physicians cannot refute, the physicians will feel criticized [35]. Of course, there are also some health care professionals trying to maintain the existing authority by not discussing OHI [45]. Guanghua [62] showed that the negative impact of $\mathrm{OHI}$ is greater, which creates greater obstacles in the communication between doctors and patients in China.

\section{OHI Seeking Affects Physician-Patient Relationships in China}

A total of 53 papers were included in this study, of which 31 $(58 \%)$ hold that $\mathrm{OHI}$ seeking has a positive impact on the physician-patient relationship, 14 (26\%) have a neutral view, and $8(15 \%)$ have a negative influence. It is worthy of in-depth study that $6(11 \%)$ papers showed that OHI seeking has a negative impact on the physician-patient relationship in China. Therefore, the negative impact of OHI on China's internet users is worthy of attention. Due to the large population of China, the time for each patient to communicate with the doctor is short, and patients choose to search online for health information more for convenience than for accuracy or authority [67]. Some studies have shown that the inclusion of some wrong medical information and reports of malignant incidents in the physician-patient relationship have a negative impact on physician-patient trust, confirming media depression theory $[67,69,70]$. Feifei [64] found that for ordinary patients, due to the professional barriers of medical knowledge, it is difficult for them to distinguish between true and false after receiving false health information on the internet. This causes patients to question doctors and leads to difficulties in the physician-patient relationship [64]. Therefore, it is important to improve patients' health information literacy and the quality of $\mathrm{OHI}$.

\section{Discussion}

\section{Principal Findings}

Based on the review of the included studies, we found that there are many factors that affect patients' choice of OHI, such as gender, age, education level, income, health literacy, and culture $[33,34,39,42,49,51]$. People with high income, education level, and health literacy are more likely to use OHI. The age difference is mainly between the young and the old. There is a digital divide between the elderly and the young [72]. One study found that older people prefer to choose people as sources of information, such as health care providers, pharmacists, relatives, and retired community workers [73]. From the perspective of patients, most of them think that OHI seeking does not affect the physician-patient relationship; some patients think it has a positive impact on the physician-patient relationship, and a few patients think it may have a negative impact on the physician-patient relationship [46]. As the impact of OHI seeking on the physician-patient relationship may be restricted by social and cultural factors, it may have adverse effects in a culture with distinct levels of patients and physicians [59].

The mechanism of $\mathrm{OHI}$ seeking affecting the physician-patient relationship is relatively complex. According to the study findings, OHI seeking can enhance patients' understanding of medical knowledge and enhance their decision-making ability and communication ability with physicians. At the same time, OHI seeking can also have an impact on patients' own psychology. Good quality of health information has a positive impact on the psychological safety of patients. Bylund [74] found that high satisfaction with OHI can promote patients' psychological security when communicating with physicians. Psychological safety has a certain impact on the distrust in the physician-patient relationship so as to affect the relationship [75]. Side effects of drugs and other information cause anxiety in patients. As the internet provides an opportunity to communicate with others about their concerns, anxiety tends to increase [76]. A small number of patients even have drug compliance changes and changes in their own medical behavior [76]. Previous studies have shown that that OHI can affect the consistency of communication between physicians and patients and the compliance of patients [33].

It is important that patients discuss health information with their physicians. Patients will seek OHI to prepare for seeing a doctor, fully participate in the decision making, and actively supplement their information during the process of seeking medical service [77]. However, this will also cause anxiety and a series of changes, such as compliance change and medical behavior change [33]. If patients do not discuss health information with physicians, the negative effects on some patients might even worsen. If patients discuss health information with physicians, these negative effects can be eliminated and alleviated. The survey results show that discussing health information with physicians is beneficial to patients' satisfaction with and trust in physicians. We must admit that patients need to discuss their health information with physicians to better promote the physician-patient relationship and improve medical services 
[78]. Good physician-patient communication can improve the clinical outcomes of some diseases [79].

Several studies have mentioned that patients are afraid to discuss health information with physicians because they are afraid of challenging the authority of physicians and even of conflicts with physicians. The OHC is not well received by the professional medical staff. They have doubts about the quality of a lot of $\mathrm{OHI}$ and whether they can explain the medical information to the patients in a better way [16]. Patients tend to remain silent if they do not feel the physician's willingness to discuss OHI with them. When patients consult about traditional and nontraditional therapies, many physicians react defensively, resulting in adverse effects on patients' trust in them and the communication between physicians and patients [80]. Some physicians try to maintain their authority as physicians by avoiding discussing OHI [45]. Due to the widespread popularity of $\mathrm{OHI}$, physicians should be aware that many patients seek $\mathrm{OHI}$ before consultation, and actively discuss and exchange $\mathrm{OHI}$ with patients [81].

Of the included 53 papers, 21 (39.6\%) studied the impact of $\mathrm{OHI}$ seeking on the physician-patient relationship in China, of which $2(9 \%)$ papers specifically mentioned that cultural factors play a potential role in $\mathrm{OHI}$ seeking for physician-patient relationships [59,62]. In the culture of hierarchical physician-patient relationships in China, the patient fully follows the physician's recommendations [59]. The popularity of OHI allows patients to play a more important role in the medical process. However, under the medical environment of "more patients, fewer physicians" in China, the communication time between each physician and patient is too short [62]. If the doctor cannot convince the patient and deny the patient's opinion directly without explanation, the conflict weakens the authority of the doctor and exacerbates the negative impact on the physician-patient relationship [62].

Several papers have shown that the internet usage time could reduce the patient's trust in the doctor [66,67]. Medical corruption, medical malpractice, physician-patient conflict, and other contents are more likely to spread among Chinese patients. Various types of negative news is frequently pushed to patients. The negative factors in the physician-patient relationship are magnified. The media often blame medical disputes on medical personnel, which exacerbates patients' distrust of doctors. In addition to negative news, the low quality of $\mathrm{OHI}$ has a negative impact on the physician-patient relationship $[61,82]$.

OHI is a double-edged sword for the relationship between physicians and patients. It is becoming increasingly important in the relationship between physicians and patients. With high-quality $\mathrm{OHI}$, it is relatively easier to have a positive impact on patients, thus promoting the physician-patient relationship. With the rapid growth and wide use of medical websites, there are important problems about the necessity of quality control [83]. The pattern of patients' access to health information is changing from passive recipients to active service seekers [77]. Health care professionals should not only discuss health information with patients but also guide them to correctly seek and use health information. Patients who can reasonably understand $\mathrm{OHI}$ can reduce the burden of physicians in the consultation and improve the communication [63].

\section{Limitations}

This study has a wide range of retrieval. When references were included, the focus was on whether health information seeking has an impact on the relationship between physicians and patients. Papers studying the impact of health information seeking on patients were not included, which may have led to missing potential research. In addition, due to the lack of a large number of studies and more reliable evidence, we could not reach a strong conclusion about how health information seeking affects the physician-patient relationship.

\section{Conclusion}

This study mainly focused on the effects of $\mathrm{OHI}$ on the relationship between physicians and patients. There are many factors influencing people's use of OHI, and young, female, highly educated, and high-income patients are more willing to search OHI. OHI seeking can affect patients' mentality and behavior. Through understanding OHI, patients can have a better understanding of medical knowledge, improve self-confidence during communication, and enhance self-decision-making behaviors. However, some OHI can lead to negative emotions and even change patients' health behaviors, due to the uneven quality of OHI. OHI seeking also affect patients' evaluation of doctors, including patients' trust in and satisfaction with physicians. OHI users choose to discuss OHI with doctors, which is beneficial to the physician-patient relationship in most cases. However, due to the subjective consciousness of patients, they may be concerned that it might affect the authority of physicians, which is the reason some patients do not initiate the discussion of health information. Moreover, the negative impact of OHI on China's internet users is worthy of attention; due to the low quality of OHI, poor health information literacy, short physician-patient communication time, and various types of negative news, patients' trust in doctors has declined. At present, China's vigorous promotion of "internet + medical health" and the reform of the hierarchical medical will be of great significance to improving the physician-patient communication model and promoting harmonious physician-patient relationships. At the same time, improving people's health information literacy and the quality of $\mathrm{OHI}$ is the crucial step in facilitating the positive effects of $\mathrm{OHI}$ on the physician-patient relationship.

\section{Acknowledgments}

The authors thank the State Key Program of the National Social Science of China (grant no. 17AZD037) and the Natural Science Foundation of Hunan Province (2019JJ80032) for their assistance in this study. We also appreciate the support of all participants. 


\section{Conflicts of Interest}

None declared.

\section{Multimedia Appendix 1}

PRISMA 2020 Checklist.

[PDF File (Adobe PDF File), 141 KB-Multimedia Appendix 1]

\section{Multimedia Appendix 2}

CASP (Critical Appraisal Skills Program) quality assessment for qualitative studies.

[PDF File (Adobe PDF File), 190 KB-Multimedia Appendix 2]

\section{Multimedia Appendix 3}

Quality assessment tool for quantitative studies.

[PDF File (Adobe PDF File), 224 KB-Multimedia Appendix 3]

\section{References}

1. Statista. Global Digital Population as of January 2021. URL: https://www.statista.com/statistics/617136/ digital-population-worldwide/ [accessed 2021-10-15]

2. $\quad$ Berger M, Wagner TH, Baker LC. Internet use and stigmatized illness. Soc Sci Med 2005 Oct;61(8):1821-1827. [doi: 10.1016/j.socscimed.2005.03.025] [Medline: 16029778]

3. Bhandari N, Shi Y, Jung K. Seeking health information online: does limited healthcare access matter? J Am Med Inform Assoc 2014;21(6):1113-1117 [FREE Full text] [doi: 10.1136/amiajnl-2013-002350] [Medline: 24948558]

4. Valero-Aguilera B, Bermúdez-Tamayo C, García-Gutiérrez JF, Jiménez-Pernett JH, Cózar-Olmo JM, Guerrero-Tejada R, et al. Information needs and internet use in urological and breast cancer patients. Support Care Cancer 2014 Feb;22(2):545-552. [doi: 10.1007/s00520-013-2009-y] [Medline: 24122406]

5. Gray NJ, Klein JD, Noyce PR, Sesselberg TS, Cantrill JA. Health information-seeking behaviour in adolescence: the place of the internet. Soc Sci Med 2005 Apr;60(7):1467-1478. [doi: 10.1016/j.socscimed.2004.08.010] [Medline: 15652680]

6. Lemire M, Paré G, Sicotte C, Harvey C. Determinants of internet use as a preferred source of information on personal health. Int J Med Inform 2008 Nov;77(11):723-734. [doi: 10.1016/j.ijmedinf.2008.03.002] [Medline: 18434246]

7. Haluza D, Naszay M, Stockinger A, Jungwirth D. Digital natives versus digital immigrants: influence of online health information seeking on the doctor-patient relationship. Health Commun 2017 Nov;32(11):1342-1349. [doi: 10.1080/10410236.2016.1220044] [Medline: 27710132]

8. Pal K, Eastwood SV, Michie S, Farmer AJ, Barnard ML, Peacock R, et al. Computer-based diabetes self-management interventions for adults with type 2 diabetes mellitus. Cochrane Database Syst Rev 2013 Mar 28(3):CD008776 [FREE Full text] [doi: 10.1002/14651858.CD008776.pub2] [Medline: 23543567]

9. Broom A. Virtually he@ @lthy: the impact of internet use on disease experience and the doctor-patient relationship. Qual Health Res 2005 Mar;15(3):325-345. [doi: 10.1177/1049732304272916] [Medline: 15761103]

10. Sommerhalder K, Abraham A, Zufferey MC, Barth J, Abel T. Internet information and medical consultations: experiences from patients' and physicians' perspectives. Patient Educ Couns 2009 Nov;77(2):266-271. [doi: 10.1016/j.pec.2009.03.028] [Medline: 19411157]

11. Jiang S, Liu J. Examining the relationship between internet health information seeking and patient-centered communication in China: taking into account self-efficacy in medical decision-making. Chin J Commun 2020 May 25;13(4):407-424. [doi: $\underline{10.1080 / 17544750.2020 .1769700]}$

12. Gantenbein L, Navarini AA, Maul LV, Brandt O, Mueller SM. Internet and social media use in dermatology patients: search behavior and impact on patient-physician relationship. Dermatol Ther 2020 Nov;33(6):e14098. [doi: 10.1111/dth.14098] [Medline: $\underline{\text { 32725746] }}$

13. Erdem SA, Harrison-Walker LJ. The role of the internet in physician-patient relationships: the issue of trust. Business Horizons 2006 Sep;49(5):387-393. [doi: 10.1016/j.bushor.2006.01.003]

14. Georgopoulou S, Prothero L, D'Cruz DP. Physician-patient communication in rheumatology: a systematic review. Rheumatol Int 2018 May 26;38(5):763-775 [FREE Full text] [doi: 10.1007/s00296-018-4016-2] [Medline: 29582095]

15. Kaba R, Sooriakumaran P. The evolution of the doctor-patient relationship. Int J Surg 2007 Feb;5(1):57-65 [FREE Full text] [doi: 10.1016/j.ijsu.2006.01.005] [Medline: 17386916]

16. Wald HS, Dube CE, Anthony DC. Untangling the web: the impact of internet use on health care and the physician-patient relationship. Patient Educ Couns 2007 Nov;68(3):218-224. [doi: 10.1016/j.pec.2007.05.016] [Medline: 17920226]

17. Emanuel EJ, Emanuel LL. Four models of the physician-patient relationship. JAMA 1992 Apr 22;267(16):2221-2226. [doi: 10.1001/jama.267.16.2221] [Medline: 1556799] 
18. National Bureau of Statistics. Bulletin of the Seventh National Census (No.2). 2021 May 11. URL: http://www.stats.gov.cn/ ztjc/zdtjgz/zgrkpc/dqcrkpc/ggl/202105/t20210519 1817695.html [accessed 2021-10-15]

19. Hong-bin X, Shou-huo J, Wen B, Yi-ming X, Xin-yi S, Zuo-fan X, et al. Integration of internet health and "Healthy China" strategy. Shanghai J Prev Med 2021;33(08):659-663. [doi: 10.19428/j.cnki.sjpm.2021.20931]

20. Si-wen L, Zhao-xia G, Shi-jun L, Xin-yi M, Bao-jia Z, Jun-xue W. Analysis on the significance and necessity of "Internet +" medical mode. China Market 2021;28(20):187-188. [doi: 10.13939/j.cnki.zgsc.2021.20.187]

21. Critical Appraisal Skills Programme. CASP Checklist: 10 Questions to Help You Make Sense of a Qualitative Research. 2018. URL: https://casp-uk.b-cdn.net/wp-content/uploads/2018/03/CASP-Qualitative-Checklist-2018 fillable form.pdf [accessed 2021-10-15]

22. Tan SS, Goonawardene N. Internet health information seeking and the patient-physician relationship: a systematic review. J Med Internet Res 2017 Jan 19;19(1):e9 [FREE Full text] [doi: 10.2196/jmir.5729] [Medline: 28104579]

23. Tian L, Hong C, Yun-jie X, Cheng-hong Z. Internet usage, physician performances and patient's trust in physician during diagnoses: investigating both pre-use and not-use internet groups. : IEEE; 2016 Presented at: 2016 49th Hawaii International Conference on System Sciences (HICSS); Jan 5-8, 2016; Koloa. [doi: 10.1109/hicss.2016.400]

24. Zhang R, Lu X, Wu W, Shang X, Liu M. Mature or emerging? The impact of treatment-related internet health information seeking on patients' trust in physicians. Int J Environ Res Public Health 2018 Aug 28;15(9):1855 [FREE Full text] [doi: 10.3390/ijerph15091855] [Medline: 30154309]

25. Xie B. Older adults' health information wants in the internet age: implications for patient-provider relationships. J Health Commun 2009 Sep;14(6):510-524. [doi: 10.1080/10810730903089614] [Medline: 19731124]

26. de Looper M, van Weert JCM, Schouten B, Bolle S, Belgers EHJ, Eddes EH, et al. The influence of online health information seeking before a consultation on anxiety, satisfaction, and information recall, mediated by patient participation: field study. J Med Internet Res 2021 Jul 05;23(7):e23670 [FREE Full text] [doi: 10.2196/23670] [Medline: 34255657]

27. Zhang Z, Yang H, He J, Lu X, Zhang R. The impact of treatment-related internet health information seeking on patient compliance. Telemed J E Health 2021 May 01;27(5):513-524. [doi: 10.1089/tmj.2020.0081] [Medline: 32907505]

28. Lu X, Zhang R. Impact of patient information behaviours in online health communities on patient compliance and the mediating role of patients' perceived empathy. Patient Educ Couns 2021 Jan;104(1):186-193. [doi: 10.1016/j.pec.2020.07.001] [Medline: 32665071]

29. Yongmei L, Xiangxi L. Effect of patient online information searching on the trust in the doctor: a cognitive dissonance theory perspective. 2019 Sep 12 Presented at: 2019 Chinese Control And Decision Conference (CCDC); June 3-5, 2019; Nanchang, China p. 4254-4259. [doi: 10.1109/ccdc.2019.8833097]

30. Zhang R, Lu X, Wu WF, Shang X. Why do patients follow physicians' advice? The influence of patients' regulatory focus on adherence: an empirical study in China. BMC Health Serv Res 2019 May 10;19(1):301 [FREE Full text] [doi: 10.1186/s12913-019-4127-9] [Medline: 31077196]

31. Peng Y, Yin PO, Deng Z, Wang R. Patient-physician interaction and trust in online health community: the role of perceived usefulness of health information and services. Int J Environ Res Public Health 2019 Dec 24;17(1):139 [FREE Full text] [doi: 10.3390/ijerph17010139] [Medline: $\underline{31878145]}$

32. Tianyue L. Study on Continuance Use Behavior of Online Healthcare Community and Its Impact on Doctor-Patient Relationship. Hefei: Hefei University of Technology; 2017. URL: https://kns.cnki.net/KCMS/detail/detail. aspx ?dbname=CMFD201702\&filename=1017179124.nh [accessed 2021-10-15]

33. Aref-Adib G, O'Hanlon P, Fullarton K, Morant N, Sommerlad A, Johnson S, et al. A qualitative study of online mental health information seeking behaviour by those with psychosis. BMC Psychiatry 2016 Jul 11;16(1):232 [FREE Full text] [doi: 10.1186/s12888-016-0952-0] [Medline: 27400874]

34. Rider T, Malik M, Chevassut T. Haematology patients and the internet: the use of on-line health information and the impact on the patient-doctor relationship. Patient Educ Couns 2014 Nov;97(2):223-238. [doi: 10.1016/j.pec.2014.06.018] [Medline: 25053474]

35. Schrank B, Sibitz I, Unger A, Amering M. How patients with schizophrenia use the internet: qualitative study. J Med Internet Res 2010 Dec 19;12(5):e70 [FREE Full text] [doi: 10.2196/jmir.1550] [Medline: 21169176]

36. Iverson S, Howard K, Penney B. Impact of internet use on health-related behaviors and the patient-physician relationship: a survey-based study and review. J Am Osteopath Assoc 2008 Dec;108(12):699-711. [doi: 10.7556/jaoa.2008.108.12.699] [Medline: 19075034]

37. Stevenson FA, Kerr C, Murray E, Nazareth I. Information from the internet and the doctor-patient relationship: the patient perspective; a qualitative study. BMC Fam Pract 2007 Aug 16;8:47 [FREE Full text] [doi: 10.1186/1471-2296-8-47] [Medline: $\underline{17705836}$ ]

38. Kivits J. Informed patients and the internet: a mediated context for consultations with health professionals. J Health Psychol 2006 Mar;11(2):269-282. [doi: 10.1177/1359105306061186] [Medline: 16464924]

39. AlGhamdi KM, Almohideb MA. Internet use by dermatology outpatients to search for health information. Int J Dermatol 2011 Mar;50(3):292-299. [doi: 10.1111/j.1365-4632.2010.04705.x] [Medline: 21342162] 
40. Silver MP. Patient perspectives on online health information and communication with doctors: a qualitative study of patients 50 years old and over. J Med Internet Res 2015 Jan 13;17(1):e19 [FREE Full text] [doi: 10.2196/jmir.3588] [Medline: 25586865]

41. Hay MC, Cadigan RJ, Khanna D, Strathmann C, Lieber E, Altman R, et al. Prepared patients: internet information seeking by new rheumatology patients. Arthritis Rheum 2008 Apr 15;59(4):575-582 [FREE Full text] [doi: 10.1002/art.23533] [Medline: 18383399$]$

42. D'Agostino TA, Ostroff JS, Heerdt A, Dickler M, Li Y, Bylund CL. Toward a greater understanding of breast cancer patients' decisions to discuss cancer-related internet information with their doctors: an exploratory study. Patient Educ Couns 2012 Oct;89(1):109-115 [FREE Full text] [doi: 10.1016/j.pec.2012.05.008] [Medline: 22722063]

43. Hay MC, Strathmann C, Lieber E, Wick K, Giesser B. Why patients go online: multiple sclerosis, the internet, and physician-patient communication. Neurologist 2008 Nov;14(6):374-381. [doi: 10.1097/NRL.0b013e31817709bb] [Medline: 19008743]

44. Murray E, Lo B, Pollack L, Donelan K, Catania J, White M, et al. The impact of health information on the internet on the physician-patient relationship: patient perceptions. Arch Intern Med 2003 Jul 28;163(14):1727-1734. [doi: 10.1001/archinte.163.14.1727] [Medline: 12885689]

45. Hart A, Henwood F, Wyatt S. The role of the Internet in patient-practitioner relationships: findings from a qualitative research study. J Med Internet Res 2004 Sep 30;6(3):e36 [FREE Full text] [doi: 10.2196/jmir.6.3.e36] [Medline: 15471762]

46. Newnham GM, Burns WI, Snyder RD, Dowling AJ, Ranieri NF, Gray EL, et al. Information from the internet: attitudes of Australian oncology patients. Intern Med J 2006 Nov;36(11):718-723. [doi: 10.1111/j.1445-5994.2006.01212.x] [Medline: $\underline{17040358]}$

47. Sillence E, Briggs P, Harris PR, Fishwick L. How do patients evaluate and make use of online health information? Soc Sci Med 2007 May;64(9):1853-1862. [doi: 10.1016/j.socscimed.2007.01.012] [Medline: 17328998]

48. Bianco A, Zucco R, Nobile CGA, Pileggi C, Pavia M. Parents seeking health-related information on the internet: cross-sectional study. J Med Internet Res 2013 Sep 18;15(9):e204 [FREE Full text] [doi: 10.2196/jmir.2752] [Medline: 24047937]

49. Chung JE. Patient-provider discussion of online health information: results from the 2007 Health Information National Trends Survey (HINTS). J Health Commun 2013;18(6):627-648. [doi: 10.1080/10810730.2012.743628] [Medline: 23590202]

50. Cocco AM, Zordan R, Taylor DM, Weiland TJ, Dilley SJ, Kant J, et al. Dr Google in the ED: searching for online health information by adult emergency department patients. Med J Aust 2018 Oct 15;209(8):342-347. [doi: 10.5694/mja17.00889] [Medline: $\underline{30107763}$ ]

51. Waring ME, McManus DD, Amante DJ, Darling CE, Kiefe CI. Online health information seeking by adults hospitalized for acute coronary syndromes: who looks for information, and who discusses it with healthcare providers? Patient Educ Couns 2018 Nov;101(11):1973-1981 [FREE Full text] [doi: 10.1016/j.pec.2018.06.016] [Medline: 30305253]

52. Russ H, Giveon SM, Catarivas MG, Yaphe J. The effect of the Internet on the patient-doctor relationship from the patient's perspective: a survey from primary care. Isr Med Assoc J 2011 Apr;13(4):220-224 [FREE Full text] [Medline: 21598810]

53. Ybarra M, Suman M. Reasons, assessments and actions taken: sex and age differences in uses of internet health information. Health Educ Res 2008 Jun;23(3):512-521. [doi: 10.1093/her/cyl062] [Medline: 16880222]

54. Chang K, Berthelet E, Grubbs E, Hamilton S, Karvat A, Tran E, et al. Websites, websites everywhere: how thyroid cancer patients use the internet. J Cancer Educ 2020 Dec;35(6):1177-1183. [doi: 10.1007/s13187-019-01576-5] [Medline: 31332622]

55. Miller MP, Arefanian S, Blatnik JA. The impact of internet-based patient self-education of surgical mesh on patient attitudes and healthcare decisions prior to hernia surgery. Surg Endosc 2020 Nov;34(11):5132-5141. [doi: 10.1007/s00464-019-07300-0] [Medline: 31832857]

56. Drug VL, Chirila I, Albusoda A, Bancila I, Iordache S, Nedelcu L, et al. How the internet influences the relationship between outpatients and gastroenterologists: a multicenter study. Turk J Gastroenterol 2020 Jan;31(1):17-22 [FREE Full text] [doi: 10.5152/tjg.2019.18353] [Medline: 31895040]

57. Lim HM, Wong SS, Yip KC, Chang FWS, Chin AJZ, Teo CH, et al. Online health information-seeking behaviour of patients attending a primary care clinic in Malaysia: a cross-sectional study. Fam Pract 2021 Aug 23:cmab099. [doi: 10.1093/fampra/cmab099] [Medline: $\underline{34423368]}$

58. Huisman M, Joye S, Biltereyst D. Searching for health: Doctor Google and the shifting dynamics of the middle-aged and older adult patient-physician relationship and interaction. J Aging Health 2020 Oct;32(9):998-1007. [doi: 10.1177/0898264319873809] [Medline: 31517558]

59. Chiu YC. Probing, impelling, but not offending doctors: the role of the internet as an information source for patients' interactions with doctors. Qual Health Res 2011 Dec;21(12):1658-1666. [doi: 10.1177/1049732311417455] [Medline: 21799204]

60. Wong DK, Cheung MK. Online health information seeking and eHealth literacy among patients attending a primary care clinic in Hong Kong: a cross-sectional survey. J Med Internet Res 2019 Mar 27;21(3):e10831 [FREE Full text] [doi: 10.2196/10831] [Medline: $\underline{30916666]}$ 
61. Hui L, Ye Z, Haiyun Q, Ying Z, Xia Q. The impact of network health information search on the relationship between doctors and patients among patients with chronic diseases. Chin Rural Health Serv Admin 2019;39(3):199-202. [doi: 10.3969/j.issn.1005-5916.2019.03.012]

62. Guanghua W. The Influence of Online Health Information Seeking Behavior on Doctor-Patient Communication. Nanjing: Nanjing University; 2018. URL: https://tinyurl.com/2p8nvaah [accessed 2021-10-15]

63. Pingping Y, Zhaohua D, Xicheng L. Study on the influence of online health information seeking on doctor-patient relationship. J Med Intell 2017;38(10):2-7,35 [FREE Full text] [doi: 10.3969/j.issn.1673-6036.2017.10.001]

64. Feifei D. Research on the Doctor-Patient Relationship under the Environment of Network. Canton: Southern Medical University; 2014. URL: https://tinyurl.com/2p8w7n98 [accessed 2021-10-15]

65. Zhijie X, Bingzhang Z, Deshuai Z, Youdong Z. The impact of the use of online health resources on the doctor-patient relationship. China Health Ind 2015;12(29):1-4. [doi: 10.16659/j.cnki.1672-5654.2015.29.001]

66. Xinjian W, Ying L. The influence of internet behavior on doctor-patient trust: based on CFPS 2016. J Northwest Norm Univ (Social Sci) 2019;56(2):119-126. [doi: 10.16783/j.cnki.nwnus.2019.02.016]

67. Bowen Z, Jiaojiang L. Will internet use affect public trust in doctors? An empirical analysis based on data CSS2013. Jiangsu Social Sci 2017(3):70-78. [doi: 10.13858/j.cnki.cn32-1312/c.2017.03.011]

68. Rui L. Research on the Influence of Online Medical Community Heaith Information Seeking Behavior on Patient Compliance. Beijing: Beijing Jiaotong University; 2019. URL: https://tinyurl.com/yckt32jk [accessed 2021-10-15]

69. Yiqun B. Influence of internet use on doctor-patient trust in the view of patients. Chin J Med Manag Sci 2021;11(04):78-83. [doi: 10.3969/j.issn.2095-7432.2021.04.017]

70. Hongze Z, Jingjing X, Suxiong W. Comparative analysis of the influence of media use on physician-patient trust and social trust. Journalism Mass Commun Mon 2017(06):68-76. [doi: 10.15897/j.cnki.cn51-1046/g2.2017.06.011]

71. Audrain-Pontevia A, Menvielle L. Effects of interpersonal trust among users of online health communities on patient trust in and satisfaction with their physician. Int J Technol Assess Health Care 2018 Feb 12;34(1):56-62. [doi: $10.1017 / \mathrm{s} 0266462317004433]$

72. Flynn KE, Smith MA, Freese J. When do older adults turn to the internet for health information? Findings from the Wisconsin Longitudinal Study. J Gen Intern Med 2006 Dec;21(12):1295-1301 [FREE Full text] [doi: 10.1111/j.1525-1497.2006.00622.x] [Medline: 16995892$]$

73. Chaudhuri S, Le T, White C, Thompson H, Demiris G. Examining health information-seeking behaviors of older adults. Comput Inform Nurs 2013 Nov;31(11):547-553 [FREE Full text] [doi: 10.1097/01.NCN.0000432131.92020.42] [Medline: 23974574]

74. Bylund CL, Gueguen JA, Sabee CM, Imes RS, Li Y, Sanford AA. Provider-patient dialogue about Internet health information: an exploration of strategies to improve the provider-patient relationship. Patient Educ Couns 2007 Jun;66(3):346-352. [doi: 10.1016/j.pec.2007.01.009] [Medline: 17329062]

75. Duckett J, Hunt K, Munro N, Sutton M. Does distrust in providers affect health-care utilization in China? Health Policy Plan 2016 Oct;31(8):1001-1009 [FREE Full text] [doi: 10.1093/heapol/czw024] [Medline: 27117483]

76. De Santis M, De Luca C, Quattrocchi T, Visconti D, Cesari E, Mappa I, et al. Use of the internet by women seeking information about potentially teratogenic agents. Eur J Obstet Gynecol Reprod Biol 2010 Aug;151(2):154-157. [doi: 10.1016/j.ejogrb.2010.04.018] [Medline: 20478650]

77. McMullan M. Patients using the internet to obtain health information: how this affects the patient-health professional relationship. Patient Educ Couns 2006 Oct;63(1-2):24-28. [doi: 10.1016/j.pec.2005.10.006] [Medline: 16406474]

78. Abdulla S, Vielhaber S, Machts J, Heinze H, Dengler R, Petri S. Information needs and information-seeking preferences of ALS patients and their carers. Amyotroph Lateral Scler Frontotemporal Degener 2014 Dec;15(7-8):505-512. [doi: 10.3109/21678421.2014.932385] [Medline: 25007829]

79. Schoenthaler A, Kalet A, Nicholson J, Lipkin M. Does improving patient-practitioner communication improve clinical outcomes in patients with cardiovascular diseases? A systematic review of the evidence. Patient Educ Couns 2014 Jul;96(1):3-12 [FREE Full text] [doi: 10.1016/j.pec.2014.04.006] [Medline: 24795073]

80. Jacob J. Consumer access to health care information: its effect on the physician-patient relationship. Alaska Med 2002;44(4):75-82. [Medline: 12650084]

81. Holland ML, Fagnano M. Appropriate antibiotic use for acute otitis media: what consumers find using Web searches. Clin Pediatr (Phila) 2008 Jun;47(5):452-456. [doi: 10.1177/0009922807313274] [Medline: 18310526]

82. Qi S, Huiling R, Yifan C, Wanjun X, Jinyin L. Survey on the status of health information use on the internet of community residents in Hefei. Med Soc 2014;27(06):62-64. [doi: 10.13723/j.yxysh.2014.06.020]

83. Neelapala P, Duvvi SK, Kumar G, Kumar BN. Do gynaecology outpatients use the internet to seek health information? A questionnaire survey. J Eval Clin Pract 2008 Apr;14(2):300-304. [doi: 10.1111/j.1365-2753.2007.00854.x] [Medline: 18324935]

\section{Abbreviations}

CNKI: China National Knowledge Infrastructure 
MESH: Medical Subject Headings

OHC: online health community

OHI: online health information

PRISMA: Preferred Reporting Items for Systematic Reviews and Meta-Analyses

Edited by G Eysenbach; submitted 10.08.20; peer-reviewed by K Lee, L Sbaffi, N Hirvonen; comments to author 21.10.20; revised version received 24.10.21; accepted 17.11.21; published 10.02.22

Please cite as:

Luo A, Qin L, Yuan Y, Yang Z, Liu F, Huang P, Xie W

The Effect of Online Health Information Seeking on Physician-Patient Relationships: Systematic Review

J Med Internet Res 2022;24(2):e23354

URL: https://www.jmir.org/2022/2/e23354

doi: $10.2196 / 23354$

PMID:

(C)Aijing Luo, Lu Qin, Yifeng Yuan, Zhengzijin Yang, Fei Liu, Panhao Huang, Wenzhao Xie. Originally published in the Journal of Medical Internet Research (https://www.jmir.org), 10.02.2022. This is an open-access article distributed under the terms of the Creative Commons Attribution License (https://creativecommons.org/licenses/by/4.0/), which permits unrestricted use, distribution, and reproduction in any medium, provided the original work, first published in the Journal of Medical Internet Research, is properly cited. The complete bibliographic information, a link to the original publication on https://www.jmir.org/, as well as this copyright and license information must be included. 